\title{
Compositional Changes to Low Water Content Bio-Oils During Aging: An NMR, GC/MS, and LC/MS Study
}

Jincy Joseph, ${ }^{\dagger}$ Matthew J. Rasmussen, ${ }^{\S}$ James P. Fecteau, ${ }^{\dagger}$ Sally Kim, ${ }^{\S}$ Hyunji Lee, ${ }^{\S}$ Katelyn A. Tracy, ${ }^{\dagger}$ Bruce L Jensen,$^{\dagger}$ Brian G. Frederick ${ }^{\dagger,+}$, Elizabeth A. Stemmler ${ }^{\dagger,},{ }^{*}$

${ }^{\dagger}$ Department of Chemistry, Forest Bioproducts Research Institute, and Laboratory for Surface Science and Technology, University of Maine, Orono, Maine 04469; ${ }^{\S}$ Department of Chemistry, Bowdoin College, Brunswick, Maine 04011

\section{Supporting Information}

Table of Contents:

\begin{tabular}{|l|r|}
\hline & Page \\
\hline Experimental Details for Quantitative Measurements & 3 \\
\hline Basis for Relating Aging Times to Effective Ambient Aging & 4 \\
\hline
\end{tabular}

Figures:

\begin{tabular}{|c|c|}
\hline $\begin{array}{l}\text { Figure SI-1. Water content for Oil A and B as a function of aging time as determined } \\
\text { with Karl Fischer titration. }\end{array}$ & 5 \\
\hline Figure SI-2. Viscosity (in kcp) of Oil B as a function of aging time. & 5 \\
\hline Figure SI-3. Structure of compounds included in this study & 6 \\
\hline $\begin{array}{l}\text { Figure SI-4. EI mass spectra of the MSTFA adducts formed with formaldehyde and } \\
\text { glycoaldehyde. }\end{array}$ & 7 \\
\hline $\begin{array}{l}\text { Figure SI-5A. Normalized abundances (mean } \pm S D ; n=3) \text { for aliphatic aldehydes and } \\
\text { furans as a function of aging time. }\end{array}$ & 8 \\
\hline $\begin{array}{l}\text { Figure SI-5B. Normalized abundances (mean } \pm S D ; n=3 \text { ) for aliphatic guaiacols as a } \\
\text { function of aging time. }\end{array}$ & 9 \\
\hline $\begin{array}{l}\text { Figure SI-5C. Normalized abundances (mean } \pm \mathrm{SD} ; \mathrm{n}=3 \text { ) for syringols as a function of } \\
\text { aging time. }\end{array}$ & 10 \\
\hline $\begin{array}{l}\text { Figure SI-5D. Normalized abundances (mean } \pm S D ; n=3) \text { for phenols, alkyl phenols, } \\
\text { and catechols as a function of aging time. }\end{array}$ & 11 \\
\hline $\begin{array}{l}\text { Figure SI-5E. Normalized abundances (mean } \pm \mathrm{SD} ; \mathrm{n}=3 \text { ) for carbohydrates (putative } \\
\text { monosaccharides) as a function of aging time. }\end{array}$ & 12 \\
\hline $\begin{array}{l}\text { Figure SI-5F. Normalized abundances (mean } \pm \mathrm{SD} ; \mathrm{n}=3 \text { ) for anhydrosugars and } \\
\text { disaccharides as a function of aging time. }\end{array}$ & 13 \\
\hline $\begin{array}{l}\text { Figure SI-5G. Normalized abundances (mean } \pm \mathrm{SD} ; \mathrm{n}=3 \text { ) for aliphatic acids and } \\
\text { hydroxyl acids as a function of aging time. }\end{array}$ & 14 \\
\hline $\begin{array}{l}\text { Figure SI-5H. Normalized abundances }(\text { mean } \pm S D ; n=3) \text { for hydroxyl ketones and } \\
\text { polyols as a function of aging time. }\end{array}$ & 15 \\
\hline $\begin{array}{l}\text { igure SI-5I. Normalized abundances (mean } \pm S D ; n=3 \text { ) for resin acids, others, and } \\
\text { ilbenes as a function of aging time. }\end{array}$ & 16 \\
\hline
\end{tabular}




\section{Tables:}

\begin{tabular}{|c|c|}
\hline Table SI-1. Gradient conditions used for LC/MS analyses & 17 \\
\hline $\begin{array}{l}\text { Table SI-2. Average molecular weight, average number, and polydispersity for Oil B } \\
\text { following accelerated aging at } 80^{\circ} \mathrm{C} \text {, as measured by gel permeation chromatography }\end{array}$ & 17 \\
\hline $\begin{array}{l}\text { Table SI-3. Oil B components detected and quantified following derivatization with } \\
\text { MSFTA and GC/MS analysis, sorted by retention time, for oils before and after } 40 \mathrm{~h} \text { of } \\
\text { aging at } 80^{\circ} \mathrm{C} \text {. }\end{array}$ & 18 \\
\hline $\begin{array}{l}\text { Table SI-4A. Concentrations of aliphatic aldehydes in Oil B before and after } 40 \mathrm{~h} \text { of } \\
\text { aging at } 80^{\circ} \mathrm{C} \text {. }\end{array}$ & 27 \\
\hline $\begin{array}{l}\text { Table SI-4B. Concentrations of hydroxy ketones and polyols in Oil B before and after } \\
40 \mathrm{~h} \text { of aging at } 80^{\circ} \mathrm{C} \text {. }\end{array}$ & 28 \\
\hline $\begin{array}{l}\text { Table SI-4C. Concentrations of aliphatic acids and hydroxyl acids in Oil B before and } \\
\text { after } 40 \mathrm{~h} \text { of aging at } 80^{\circ} \mathrm{C} \text {. }\end{array}$ & 28 \\
\hline $\begin{array}{l}\text { Table SI-4D. Concentrations of phenols in Oil B before and after } 40 \mathrm{~h} \text { of aging at } 80 \\
{ }^{\circ} \mathrm{C} \text {. }\end{array}$ & 29 \\
\hline $\begin{array}{l}\text { Table SI-4E. Concentrations of alkyl-substituted catechols in Oil B before and after } 40 \\
\text { h of aging at } 80^{\circ} \mathrm{C} \text {. }\end{array}$ & 29 \\
\hline $\begin{array}{l}\text { Table SI-4F. Concentrations of hydroxybenzenes in Oil B before and after } 40 \mathrm{~h} \text { of } \\
\text { aging at } 80^{\circ} \mathrm{C} \text {. }\end{array}$ & 30 \\
\hline Table SI-4G. Concentrations of furans in Oil B before and after $40 \mathrm{~h}$ of aging at $80^{\circ} \mathrm{C}$. & 30 \\
\hline $\begin{array}{l}\text { Table SI-4H. Concentrations of guaiacols in Oil B before and after } 40 \mathrm{~h} \text { of aging at } 80 \\
{ }^{\circ} \mathrm{C} .\end{array}$ & 31 \\
\hline $\begin{array}{l}\text { Table SI-4I. Concentrations of syringols in Oil B before and after } 40 \mathrm{~h} \text { of aging at } 80 \\
{ }^{\circ} \mathrm{C} \text {. }\end{array}$ & 32 \\
\hline $\begin{array}{l}\text { Table SI-4J. Concentrations of sugars and anhydrosugars in Oil B before and after } 40 \mathrm{~h} \\
\text { of aging at } 80{ }^{\circ} \mathrm{C} \text {. }\end{array}$ & 33 \\
\hline $\begin{array}{l}\text { Table SI-4K. Concentrations of resin acids in Oil B before and after } 40 \mathrm{~h} \text { of aging at } 80 \\
{ }^{\circ} \mathrm{C} \text {. }\end{array}$ & 34 \\
\hline $\begin{array}{l}\text { Table SI-4L. Concentrations of stilbenes in Oil B before and after } 40 \mathrm{~h} \text { of aging at } 80 \\
{ }^{\circ} \mathrm{C} \text {. }\end{array}$ & 34 \\
\hline Table SI-4M. Concentrations of others in Oil B before and after $40 \mathrm{~h}$ of aging at $80^{\circ} \mathrm{C}$. & 34 \\
\hline $\begin{array}{l}\text { Table SI-5. Summary of exact mass measurements for a putative dihydroxybenzene } \\
\text { dimer, detected as the peracetylated form at } 30.9 \mathrm{~min} \text { in the chip-nanoESI-Q-TOF } \\
\text { MS/MS analysis of Oil B. }\end{array}$ & 35 \\
\hline
\end{tabular}




\section{Experimental Details for Quantitative Measurements}

Compounds were identified using standards (S), library (Lib) or literature spectra (Lit), or mass spectral interpretation (I). Quantitative analysis of oil components was conducted using response factor from standards $(\mathrm{S})$ or using response factors based upon structurally related compounds (SR). For response factors based on structurally related compounds, compound that were chromatographically resolved were quantified using the integrated total ionization chromatogram (TIC) peak area for both the standard and analyte (SR-TIC); for compounds that co-eluted with other bio-oil components, the peak area for an ion specific to the analyte was integrated. This area was adjusted to give the corresponding TIC area using the contribution of the selected ion to the total ionization signal (SR-EIC). Standards for some components could not be used reliably for quantification because the components were unstable or formed multiple products upon derivatization $\left(\mathrm{S}^{*}\right)$. Concentrations reported with an asterisk should be considered semi-quantitative for that reason.

Kinetic measurements, shown in Figures SI-5A-I, were made using EICs for all analytes and the peak ratio relative to the internal standard, biphenyl was calculated. For all compounds, we assess relative changes as a function of aging time by normalizing the data to the most abundant signal. 


\section{Basis for Relating Aging Times to Effective Ambient Aging}

Czernik, et al. ${ }^{1}$ measured water content, viscosity, and molecular weight changes as a function of time at 37,60 , and $90^{\circ} \mathrm{C}$ and showed that the changes in viscosity and weight-average molecular weight could be described by a first order reaction law, where the conversion, $\alpha_{t}=$ $\left(M_{t}-M_{0}\right) /\left(M_{\infty}-M_{0}\right)$ fits the integrated rate law, $-\ln (1-\alpha)=k t$, where the rate constant, $k=\operatorname{Aexp}\left(-E_{a} / R T\right)$. They obtained an activation energy, $\mathrm{E}_{\mathrm{a}}=102 \pm 11 \mathrm{~kJ} / \mathrm{mol}$, for an oak derived fast pyrolysis oil. Therefore, the rate of the collective aging reactions can be approximated, within a first order process, as $r_{T}=A \exp \left(-\frac{E_{a}}{R T}\right)[M]_{t}$. Therefore, the relative rates of the aging reaction at temperature, $\mathrm{T}$, compared to ambient temperature, $\mathrm{T}_{\mathrm{amb}}$, can be estimated as $\frac{r_{T}}{r_{a m b}}=$

$\exp \left(-E_{a} / R\left[\frac{1}{T}-\frac{1}{T_{a m b}}\right]\right)$. The effective aging time is the relative rate times the actual aging time at elevated temperature.

The relative rates at temperature $\mathrm{T}$ and room temperature, $20.6^{\circ} \mathrm{C}$, based on recent work by Elliot, et al. ${ }^{2}$, where aging for $24 \mathrm{~h}$ at $80{ }^{\circ} \mathrm{C}$ correspond to $6 \mathrm{mo}$ at room temperature implies that the aging rate is 180 times faster at $80^{\circ} \mathrm{C}$ and the activation energy is $75.6 \mathrm{~kJ} / \mathrm{mol}$.

Using this approach, we estimate that $40 \mathrm{~h}$ at $80{ }^{\circ} \mathrm{C}$ corresponds to 10 months at $20.6^{\circ} \mathrm{C}$. The relative rate increase to 356 at $90^{\circ} \mathrm{C}$, so aging for $32 \mathrm{~h}$ would correspond to about 15.8 months, which agrees well with estimates by Brown (private communication) based on their studies ${ }^{3}$ at 90 ${ }^{\circ} \mathrm{C}$ where 8 hours correspond to approximately 4 months, although they used a second order rate law to model the polymerization processes.

Clearly, both kinetic factors and experimental aspects of reproducibly defining an accelerated aging procedure ${ }^{2}$ limit the accuracy of the effective aging time and estimates should be regarded as qualitative.

\section{References}

1. Czernik, S.; Johnson, D. K.; Black, S., Stability of wood fast pyrolysis oil. Biomass Bioenergy 1994, 7 (1-6), 187-92.

2. Elliott, D. C.; Oasmaa, A.; Meier, D.; Preto, F.; Bridgwater, A. V., Results of the IEA round robin on viscosity and aging of fast pyrolysis bio-oils: long-term tests and repeatability. Energy Fuels 2012, 26 (12), 7362-7366.

3. Brown, R. C.; Meyer, T.; Fox, R.; Submramaniam, S.; Shanks, B.; Smith, R. G. A Systems Approach to Bio-Oil Stabilization - Final Technical Report; DOE/GO18205; 2011; pp 1-101. 


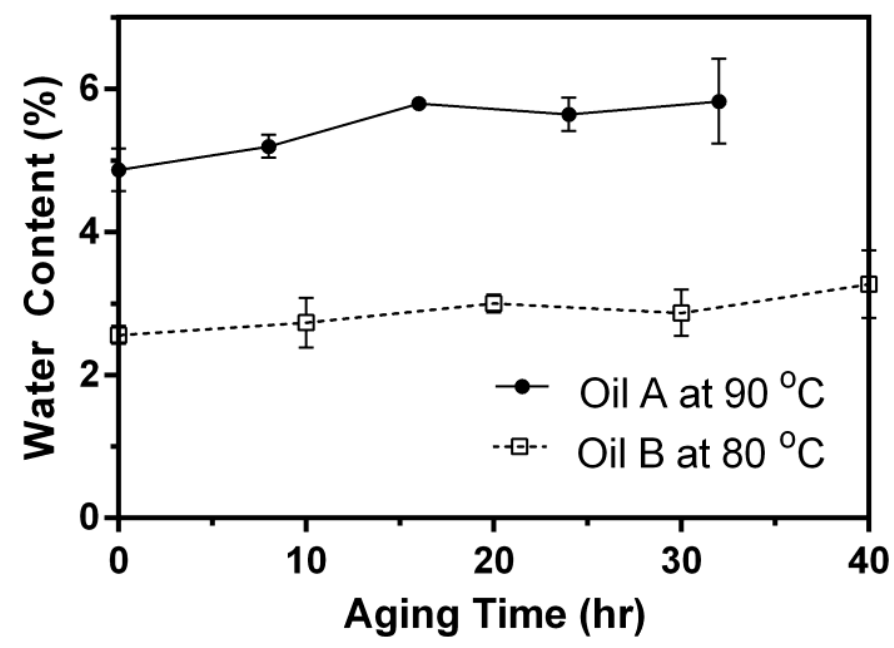

Figure SI-1. Water content for Oil A and B as a function of aging time as determined with Karl Fischer titration. Pine ESP pyrolysis Oil A was aged at $90^{\circ} \mathrm{C}$ for intervals of $8 \mathrm{~h}$, corresponding to an effective ambient aging time of 16 months, and Oil B aged at $80^{\circ} \mathrm{C}$ for intervals of $10 \mathrm{~h}$, corresponding to an effective aging time of 10 months. Measurements were made in triplicate; error bars represent the $95 \%$ confidence intervals.

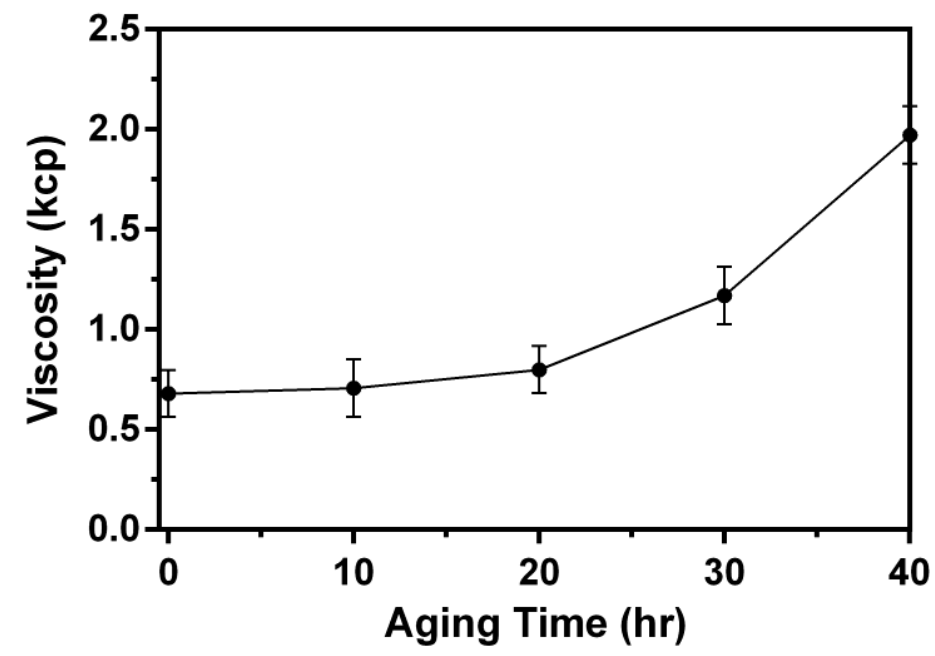

Figure SI-2. Viscosity (in kcp) of Oil B as a function of aging time at $80^{\circ} \mathrm{C}$, corresponding to an effective ambient aging time of 10 months. Error bars represent the $95 \%$ confidence intervals. 

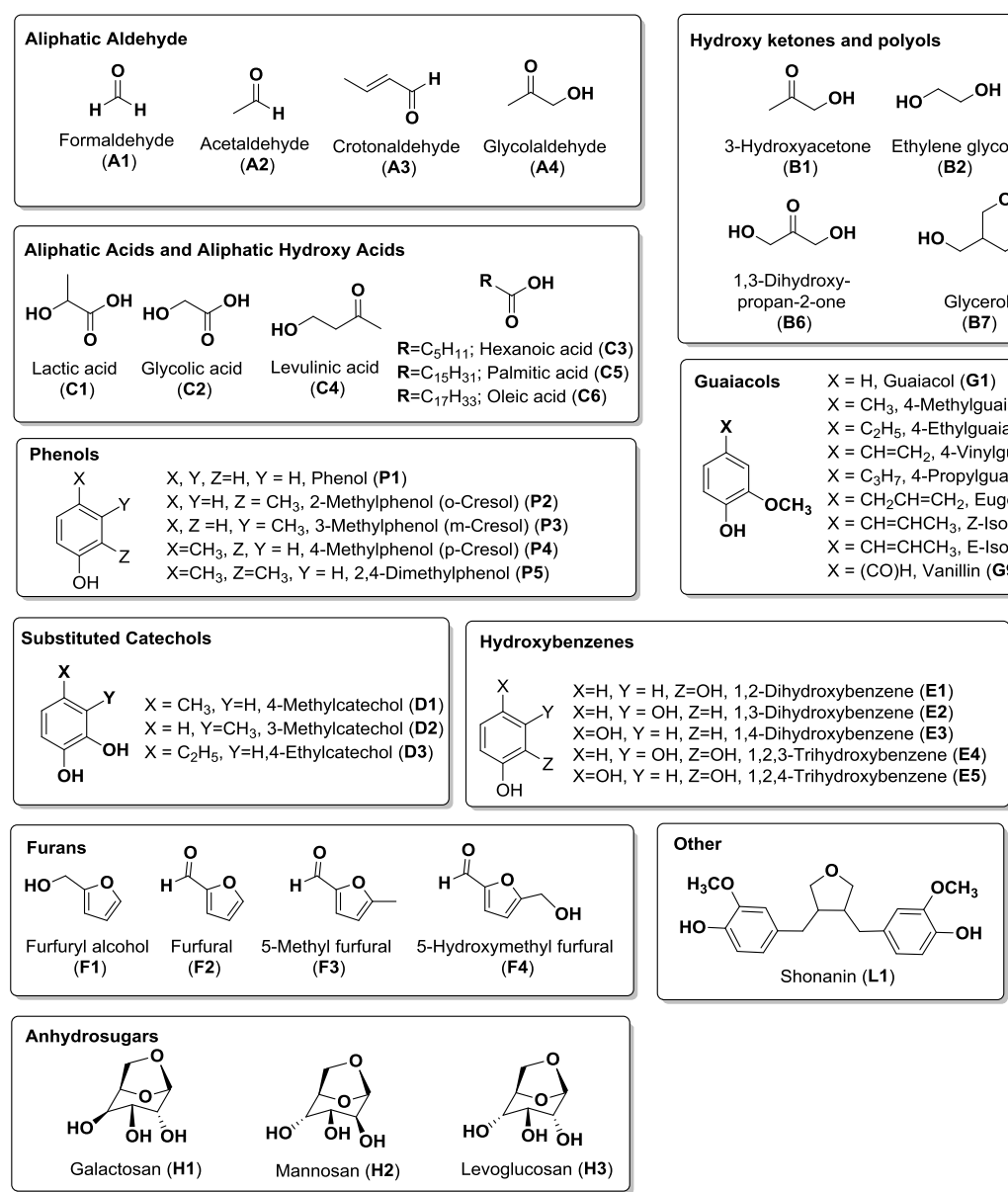

(1)
$X, Y, Z=\mathrm{H}, \mathrm{Y}=\mathrm{H}$, Phenol (P1)
$\mathrm{X}, \mathrm{Y=H}, \mathrm{Z}=\mathrm{CH}_{3}$, 2-Methylphenol (o-Cresol) (P2)
$\mathrm{X}, \mathrm{Z}=\mathrm{H}, \mathrm{Y}=\mathrm{CH}_{3}$, 3-Methylphenol (m-Cresol) (P3)
$X=\mathrm{CH}_{3}, \mathrm{Z}, \mathrm{Y}=\mathrm{H}, 4$-Methylphenol (p-Cresol) (P4)
$X=\mathrm{CH}_{3}, \mathrm{Z}=\mathrm{CH}_{3}, \mathrm{Y}=\mathrm{H}$, 2,4-Dimethylphenol (P5)

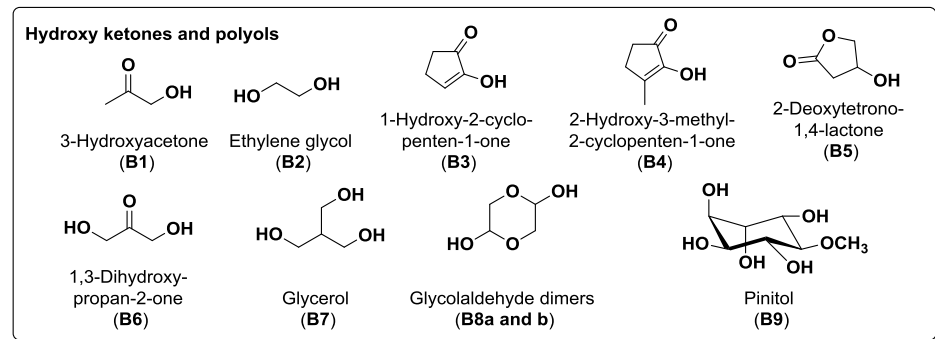

\begin{tabular}{|c|c|c|}
\hline \multirow[t]{2}{*}{ Guaiacols } & $X=H$, Guaiacol (G1) & \\
\hline & $\mathrm{X}=\mathrm{CH}_{3}$, 4-Methylguaiacol (G2) & $\mathrm{X}=(\mathrm{CO}) \mathrm{CH}_{3}$, Acetovanillone $(\mathbf{G} 10)$ \\
\hline $\mathrm{x}$ & $\mathrm{X}=\mathrm{C}_{2} \mathrm{H}_{5}, 4$-Ethylguaiacol $(\mathbf{G} \mathbf{3})$ & $\mathrm{X}=\mathrm{CH}_{2}(\mathrm{CO}) \mathrm{CH}_{3}$, Guaiacylacetone (G11) \\
\hline & $\mathrm{X}=\mathrm{CH}=\mathrm{CH}_{2}, 4$-Vinylguaiacol $(\mathbf{G} \mathbf{4})$ & $\mathrm{X}=\mathrm{CH}_{2} \mathrm{CH}_{2} \mathrm{OH}$, Homovanillyl alcohol $(\mathbf{G} 12)$ \\
\hline & $\mathrm{X}=\mathrm{C}_{3} \mathrm{H}_{7}$, 4-Propylguaiacol (G5) & $\mathrm{X}=\mathrm{CH}_{2} \mathrm{CH}_{2} \mathrm{CO}_{2} \mathrm{H}$, Vanillic acid $(\mathbf{G} 13)$ \\
\hline $\mathrm{CH}_{3}$ & $\mathrm{X}=\mathrm{CH}_{2} \mathrm{CH}=\mathrm{CH}_{2}$, Eugenol (G6) & $\mathrm{X}=\mathrm{CH}_{2} \mathrm{CH}_{2} \mathrm{CH}_{2} \mathrm{OH}$, 3-Vanilpropanol (G14) \\
\hline$n_{3}$ & $\mathrm{X}=\mathrm{CH}=\mathrm{CHCH}_{3}$, Z-Isoeugenol $(\mathbf{G} 7)$ & $\mathrm{X}=\mathrm{CH}=\mathrm{CHCH}_{2} \mathrm{OH}, \mathrm{Z}$-Coniferylalcohol (G15) \\
\hline & $\mathrm{X}=\mathrm{CH}=\mathrm{CHCH}_{3}$, E-Isoeugenol $(\mathbf{G} 8)$ & $\mathrm{X}=\mathrm{CH}=\mathrm{CHCH}_{2} \mathrm{OH}$, E-Coniferylalcohol (G16) \\
\hline & $\mathrm{X}=(\mathrm{CO}) \mathrm{H}$, Vanillin $(\mathbf{G 9})$ & $\mathrm{X}=\mathrm{CH}=\mathrm{CH}(\mathrm{CO}) \mathrm{H}$, Coniferylaldehyde $(\mathbf{G} 17)$ \\
\hline
\end{tabular}

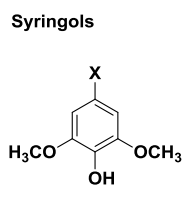

$\mathrm{X}=\mathrm{H}$, Syringol $(\mathbf{S} \mathbf{1})$

$\mathrm{X}=\mathrm{CH}_{3}$, 4-Methylsyringol $(\mathbf{S} 2)$

$\mathrm{X}=\mathrm{C}_{2} \mathrm{H}_{5}, 4$-Ethylsyringol $(\mathbf{S} 3)$

$\mathrm{X}=\mathrm{CH}=\mathrm{CH}_{2}, 4$-Vinylsyringol (S4)

$\mathrm{X}=\mathrm{CH}_{2} \mathrm{CH}=\mathrm{CH}_{2}, 4-$ Ethenylsyringol (S5)

$\mathrm{X}=\mathrm{CH}=\mathrm{CHCH}_{3}$, 4-Propenylsyringol- $\mathrm{Z}$ (S6)

$\mathrm{X}=\mathrm{CH}=\mathrm{CHCH}_{3}$, 4-Propenylsyringol- $E(\mathbf{S 7})$

CO) $\mathrm{H}$, Syringaldehyde (S8)

$\mathrm{X}=\mathrm{CH}=\mathrm{CH}(\mathrm{CO}) \mathrm{H}$, Sinapaldehyde $(\mathbf{S 9})$
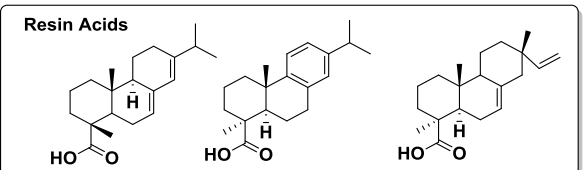

Abietic acid (J1) Dehydroabietic acid (J2) Isopimaric acid (J3)

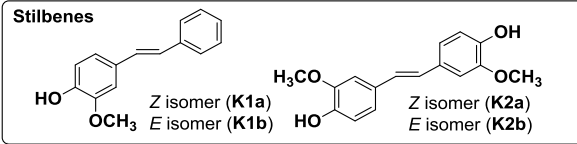

Figure SI-3. Structure of compounds included in this study 

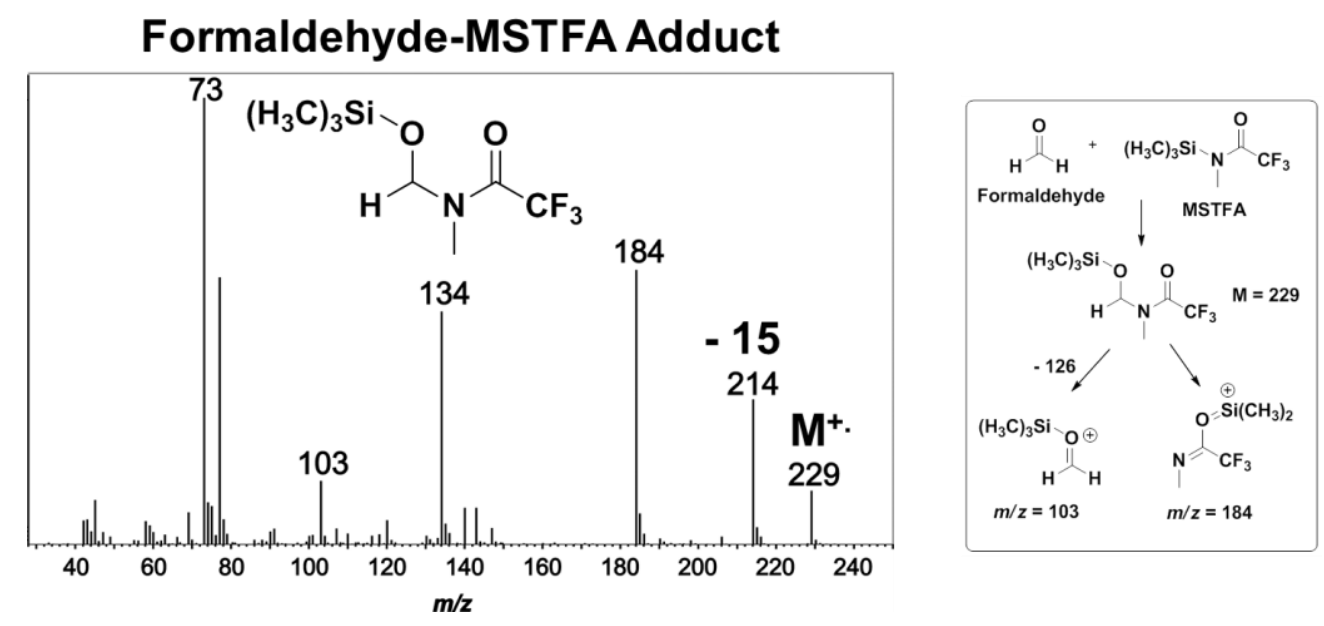

\section{Glycoaldehyde-MSTFA Adduct}
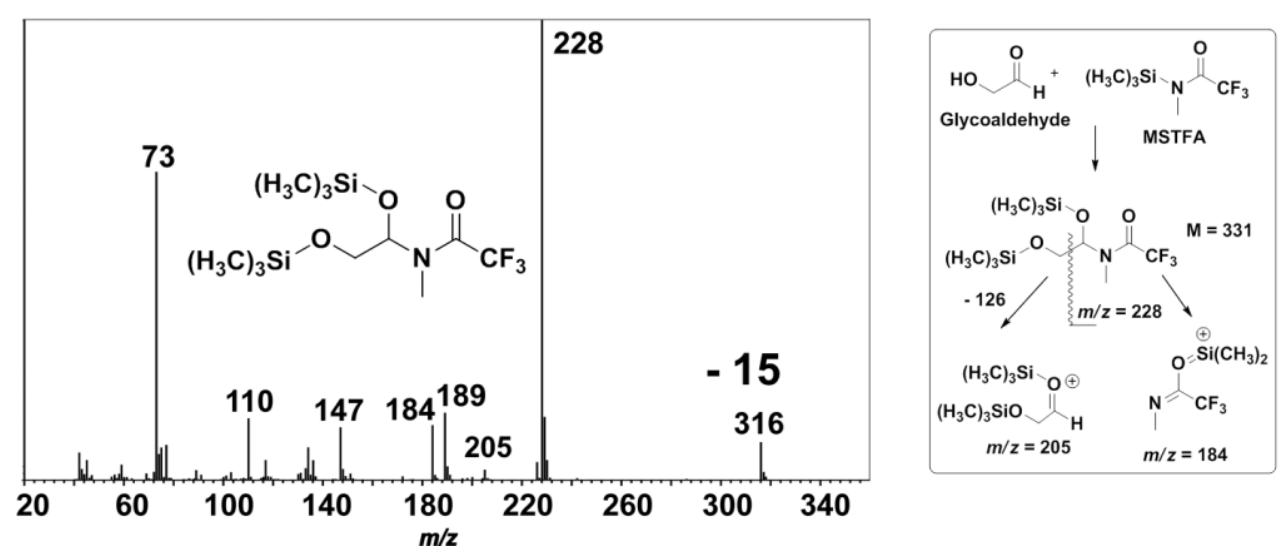

Figure SI-4. EI mass spectra of the MSTFA adducts formed with formaldehyde and glycoaldehyde. 


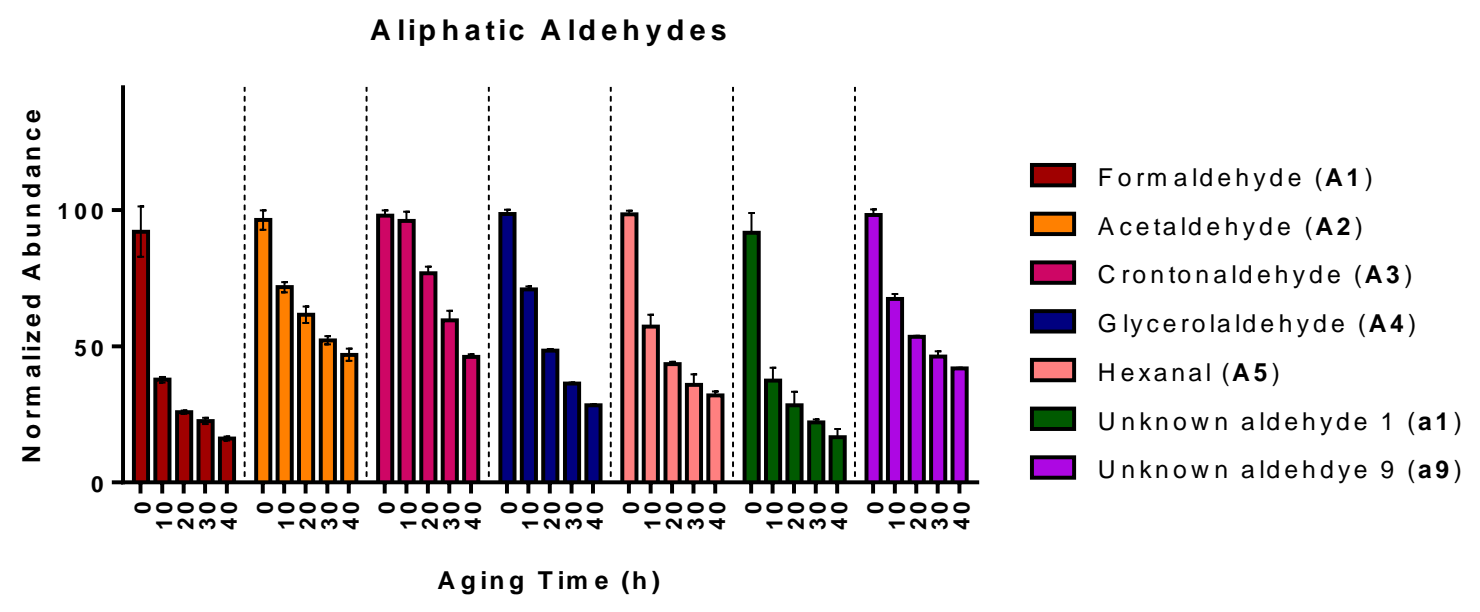

Furans

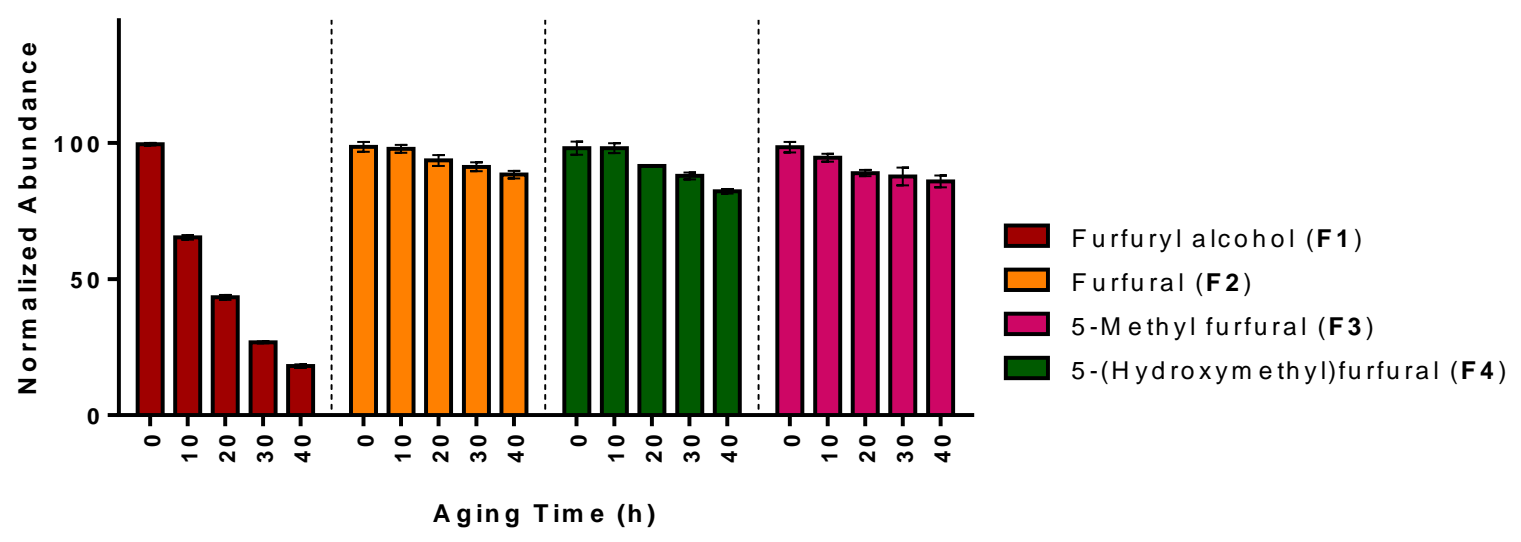

Figure SI-5A. Normalized abundances (mean $\pm \mathrm{SD} ; \mathrm{n}=3$ ) for aliphatic aldehydes and furans as a function of aging time at $80{ }^{\circ} \mathrm{C}$. 
Guaiacols I

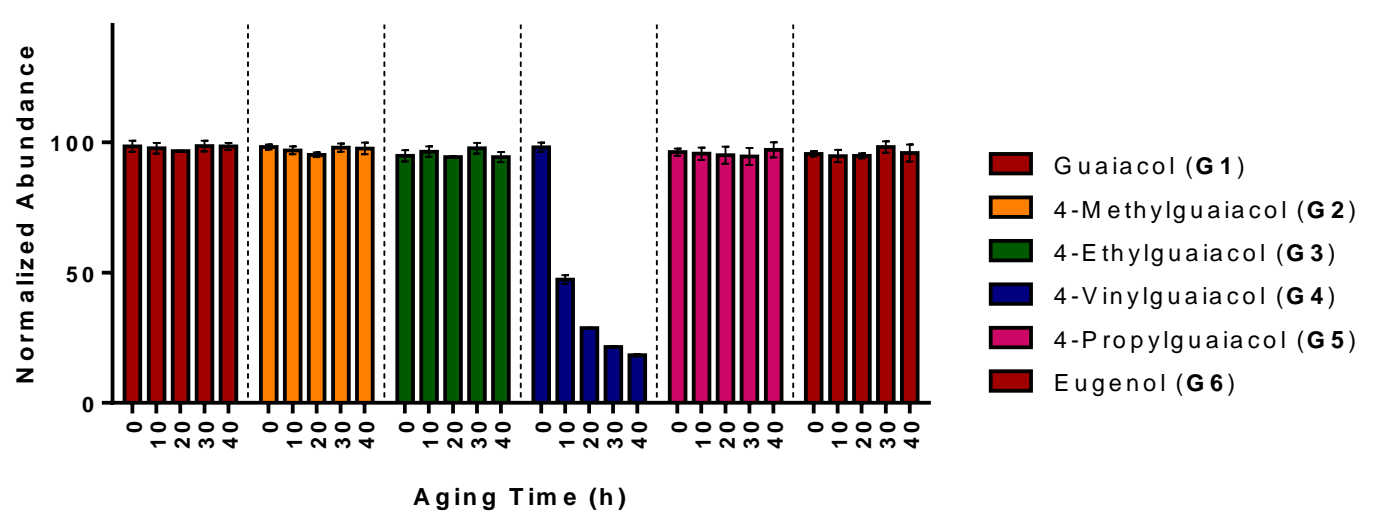

Guaiacols II

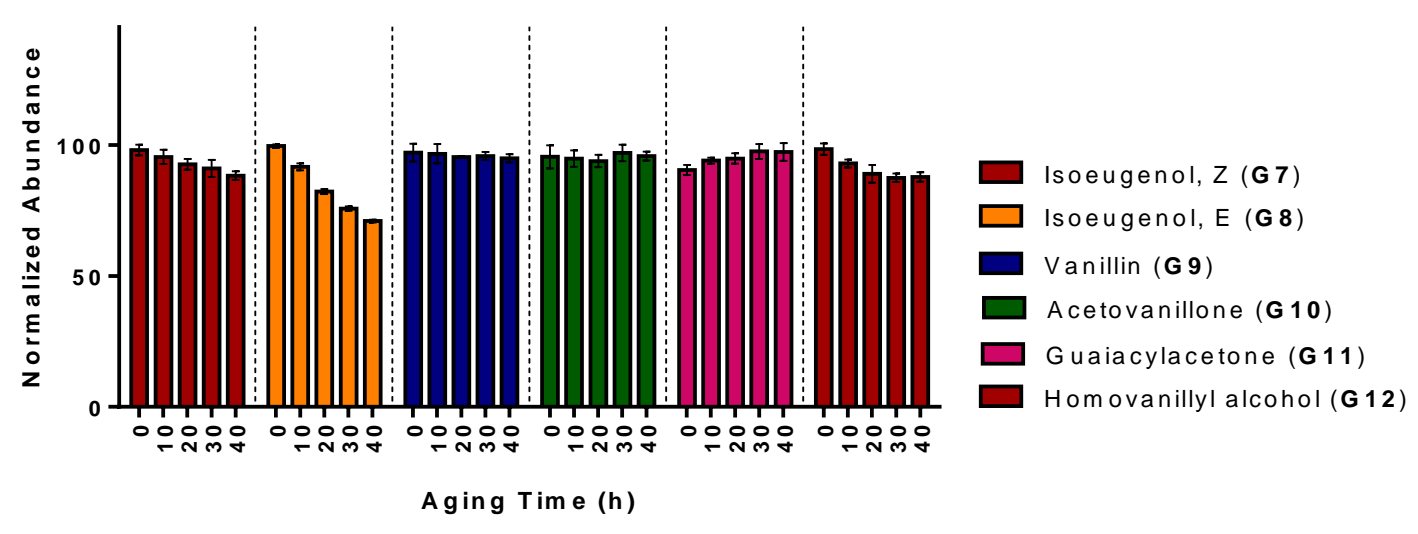

Guaiacols III

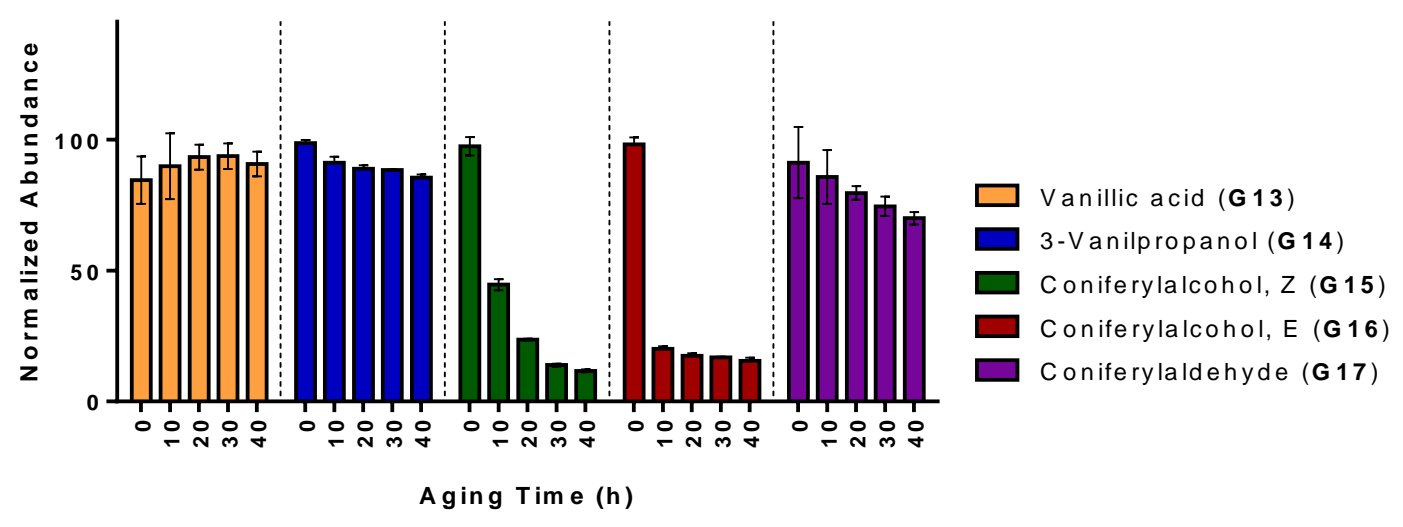

Figure SI-5B. Normalized abundances (mean $\pm \mathrm{SD} ; \mathrm{n}=3$ ) for guaiacols as a function of aging time at $80{ }^{\circ} \mathrm{C}$. 

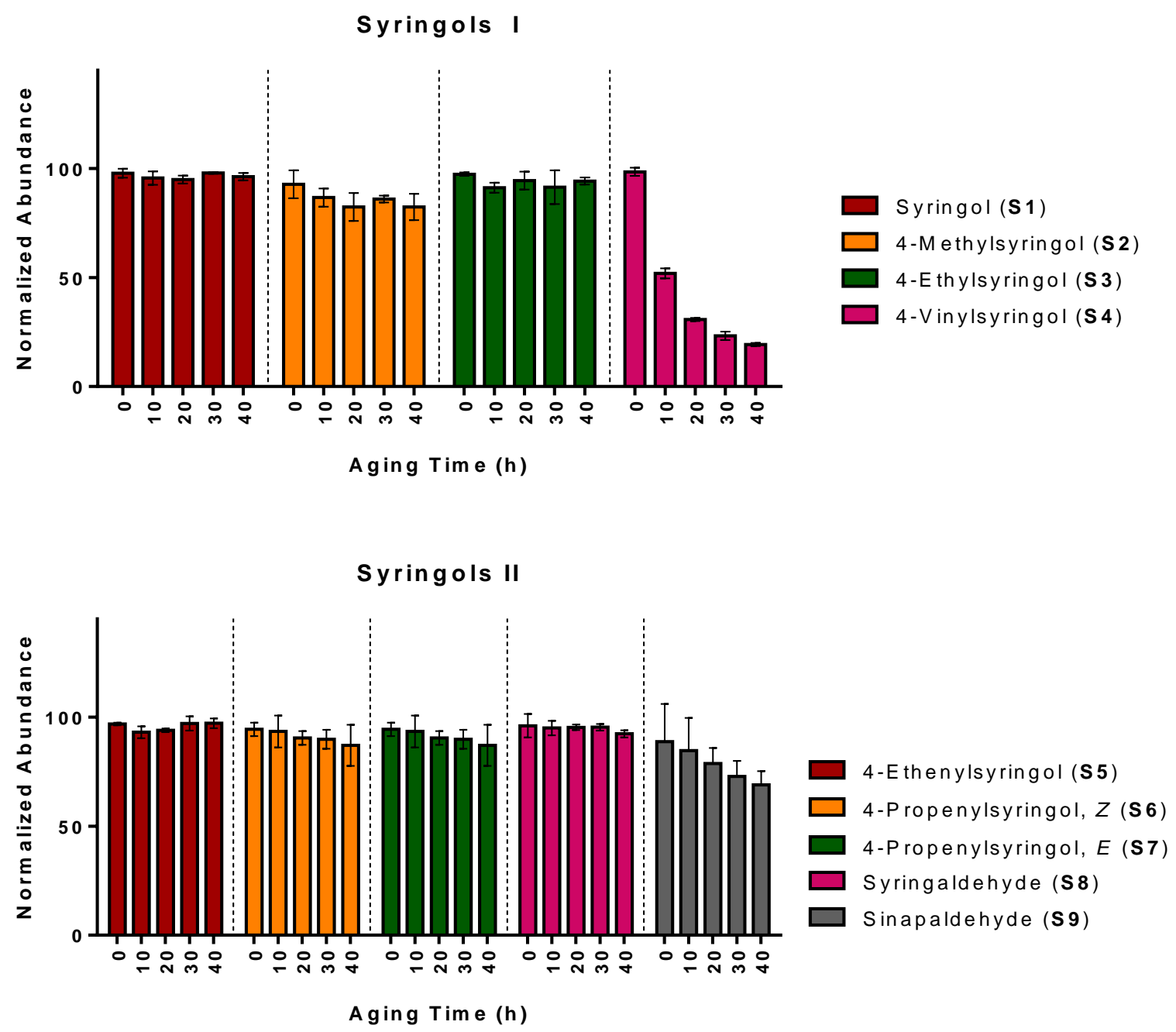

Figure SI-5C. Normalized abundances (mean $\pm \mathrm{SD} ; \mathrm{n}=3$ ) for syringols as a function of aging time at $80{ }^{\circ} \mathrm{C}$. 

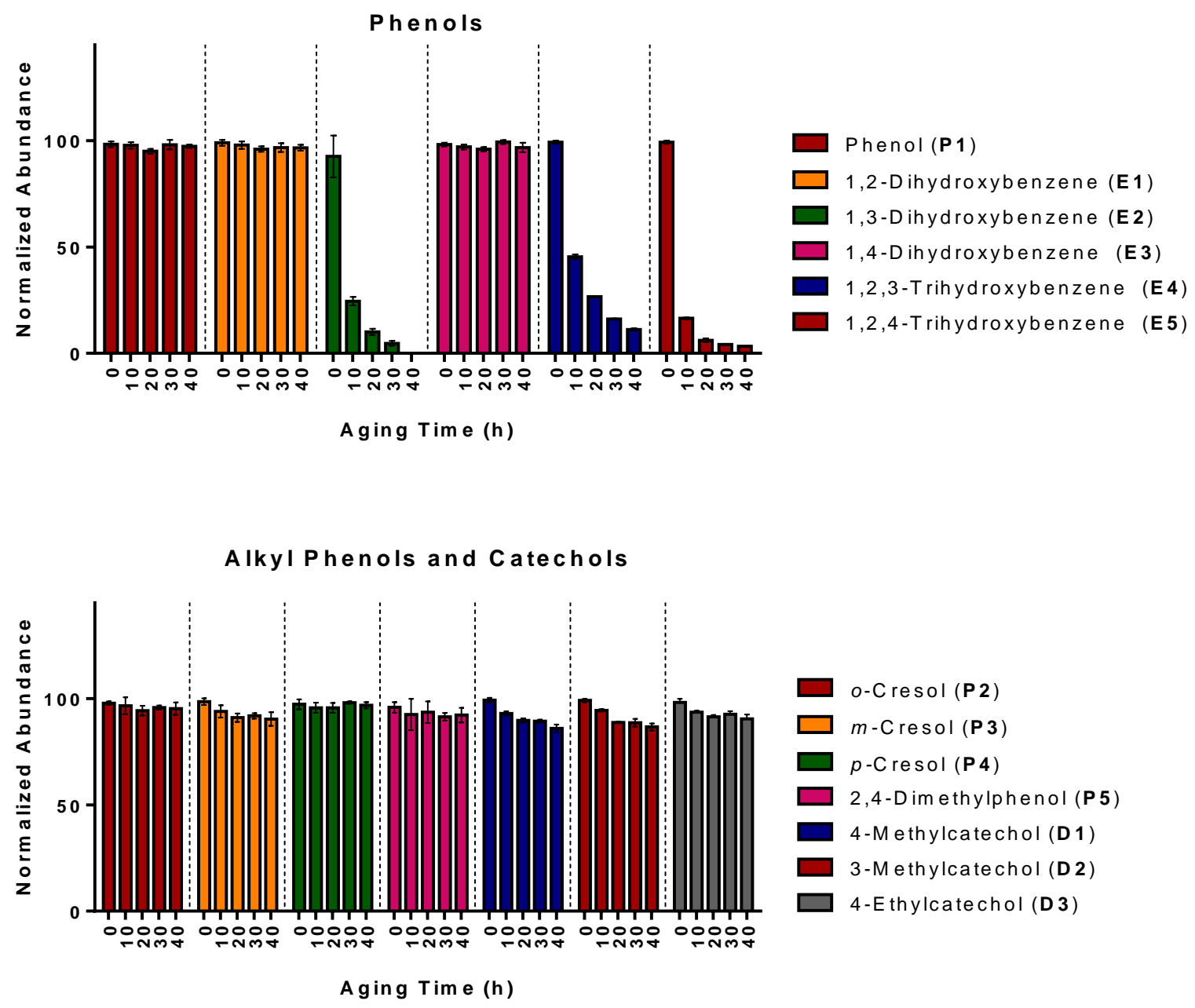

Figure SI-5D. Normalized abundances (mean $\pm \mathrm{SD} ; \mathrm{n}=3$ ) for phenols, alkyl phenols, and catechols as a function of aging time at $80{ }^{\circ} \mathrm{C}$. 

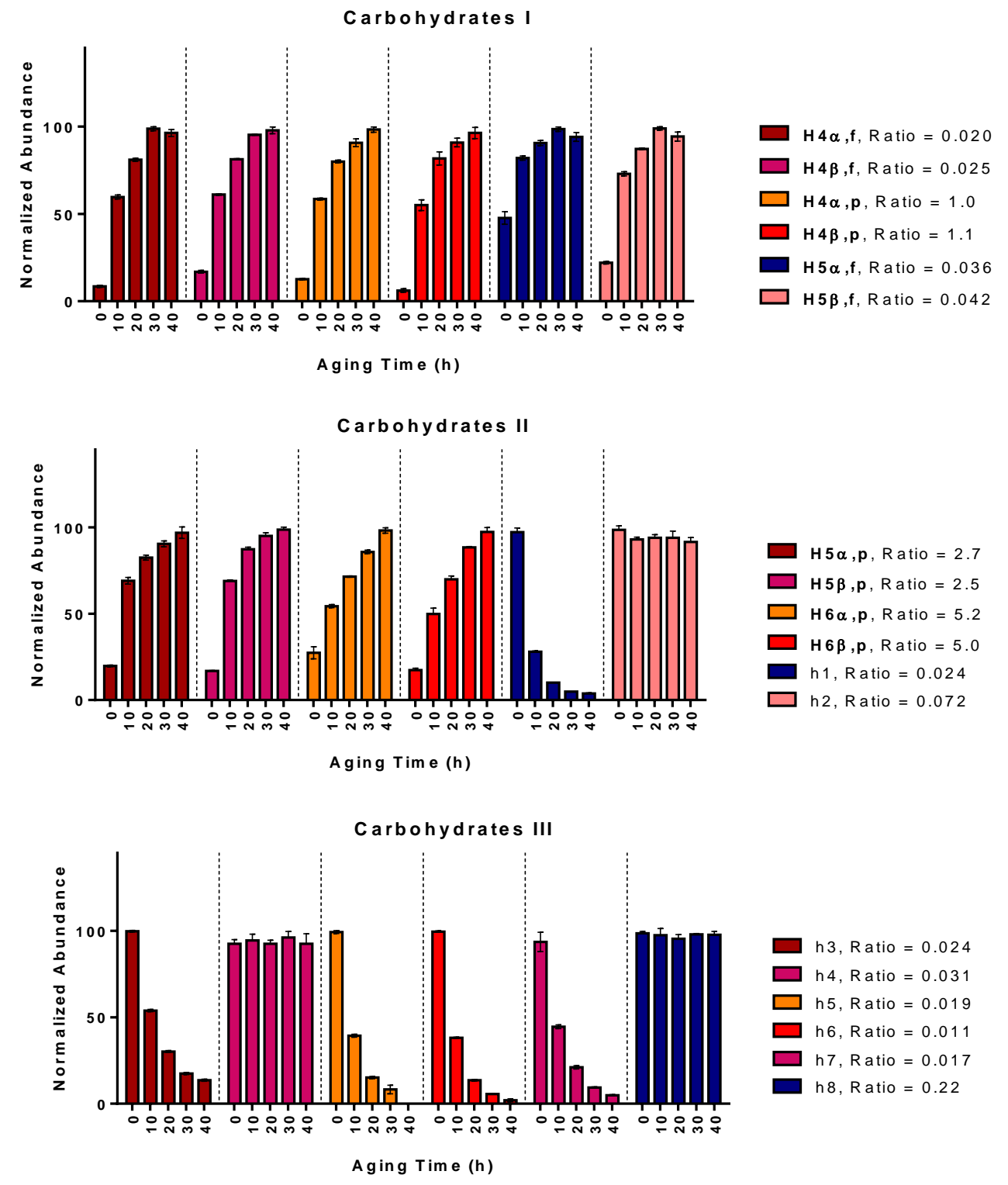

Figure SI-5E. Normalized abundances (mean $\pm \mathrm{SD} ; \mathrm{n}=3$ ) for carbohydrates (putative monosaccharides) as a function of aging time at $80{ }^{\circ} \mathrm{C}$. For the sugars $\mathrm{H} 4$ (arabinose), $\mathrm{H} 5$ (xylose), and H6 (glucose) the " $\alpha$ " refers to the alpha-anomer, the " $\beta$ " refers to the beta-anomer, the "f" refers to furanose-ring form of the monosaccharide, and the "p" refers to the pyranosering form of the monosaccharide. Compounds symbolized with a lower case " $h$ " have not been identified; "Ratio" is the ratio of the $m / z 204$ ion to the $m / z 217$ ion. Furano-ring sugars are characterized by low ratios; pyrano-ring sugars are characterized by high ratios (generally greater than 1). 

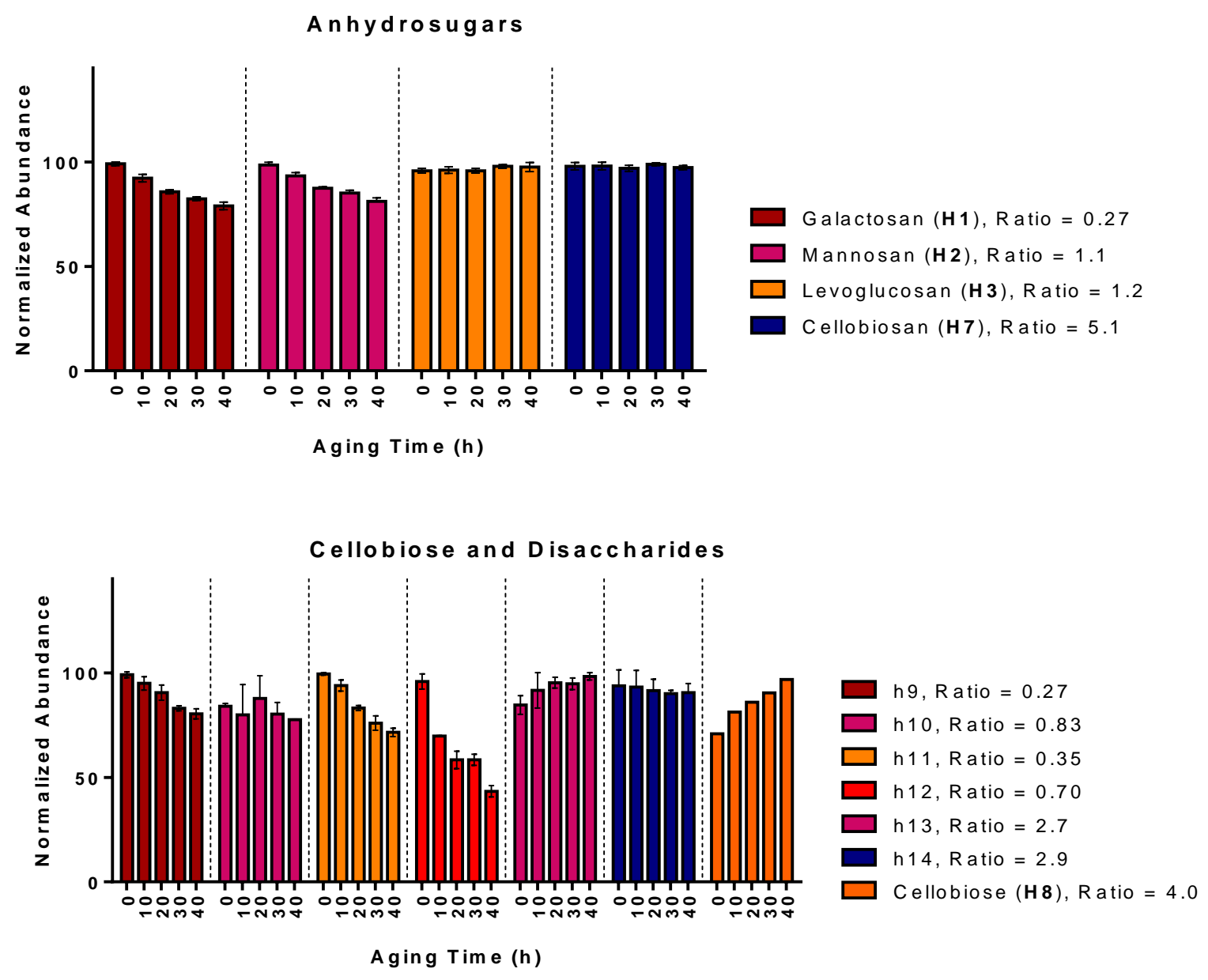

Figure SI-5F. Normalized abundances (mean $\pm S D ; n=3$ ) for anhydrosugars and other putative disaccharide carbohydrates as a function of aging time at $80{ }^{\circ} \mathrm{C}$. Compounds symbolized with a lower case "h" have not been identified; "Ratio" is the ratio of the $m / z 204$ ion to the $m / z 217$ ion. Furano-ring sugars are characterized by low ratios; pyrano-ring sugars are characterized by high ratios (generally greater than 1 ). 


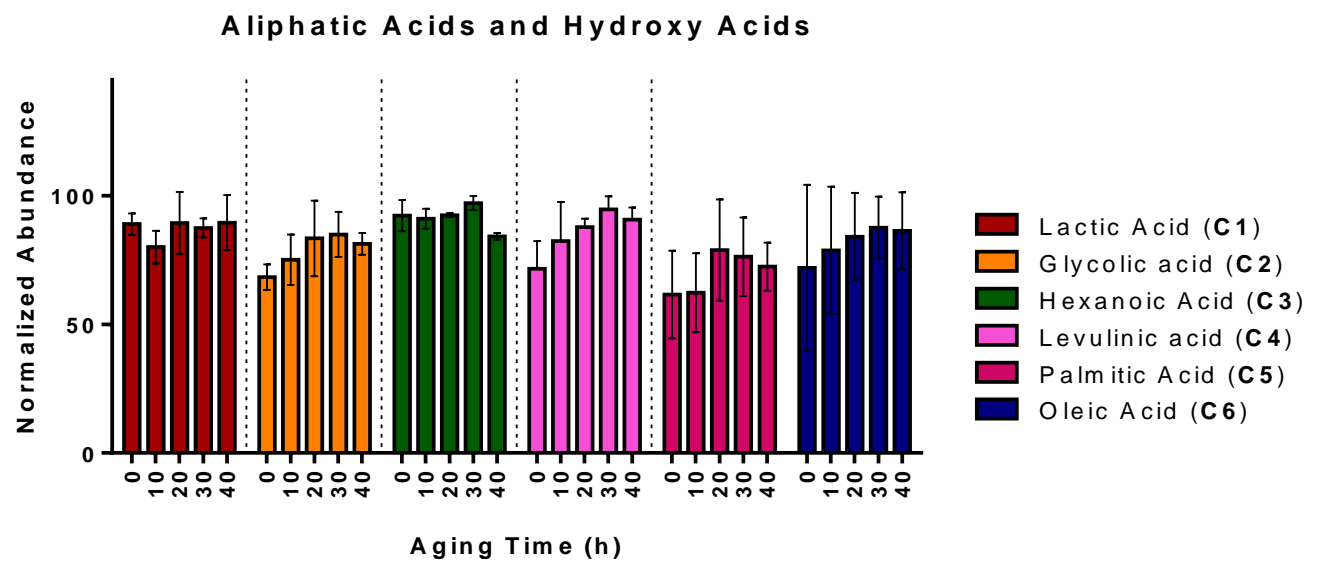

Figure SI-5G. Normalized abundances (mean $\pm \mathrm{SD} ; \mathrm{n}=3$ ) for aliphatic acids and hydroxyl acids as a function of aging time at $80^{\circ} \mathrm{C}$. 

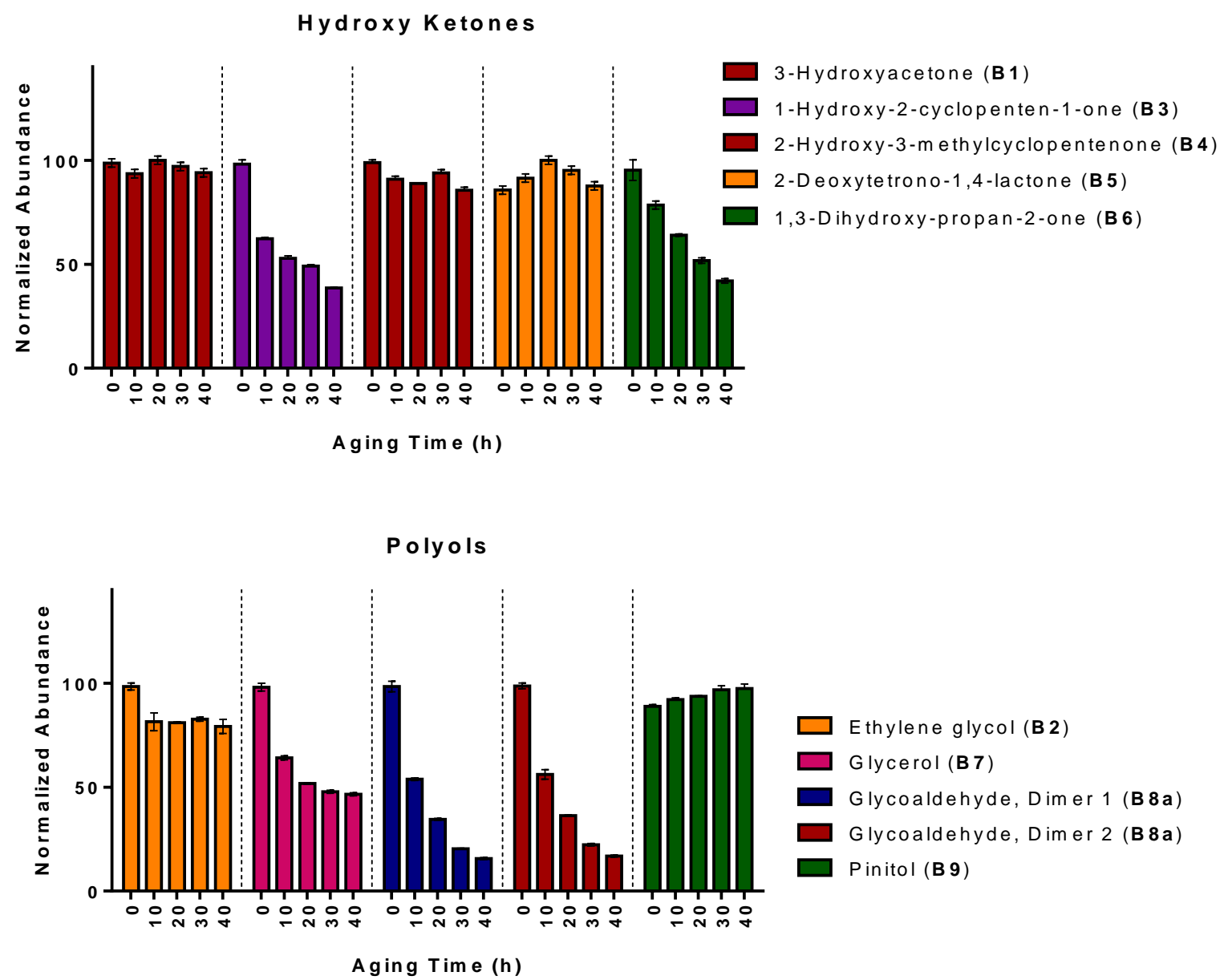

Figure SI-5H. Normalized abundances (mean $\pm \mathrm{SD} ; \mathrm{n}=3$ ) for hydroxyl ketones and polyols as a function of aging time at $80^{\circ} \mathrm{C}$. 

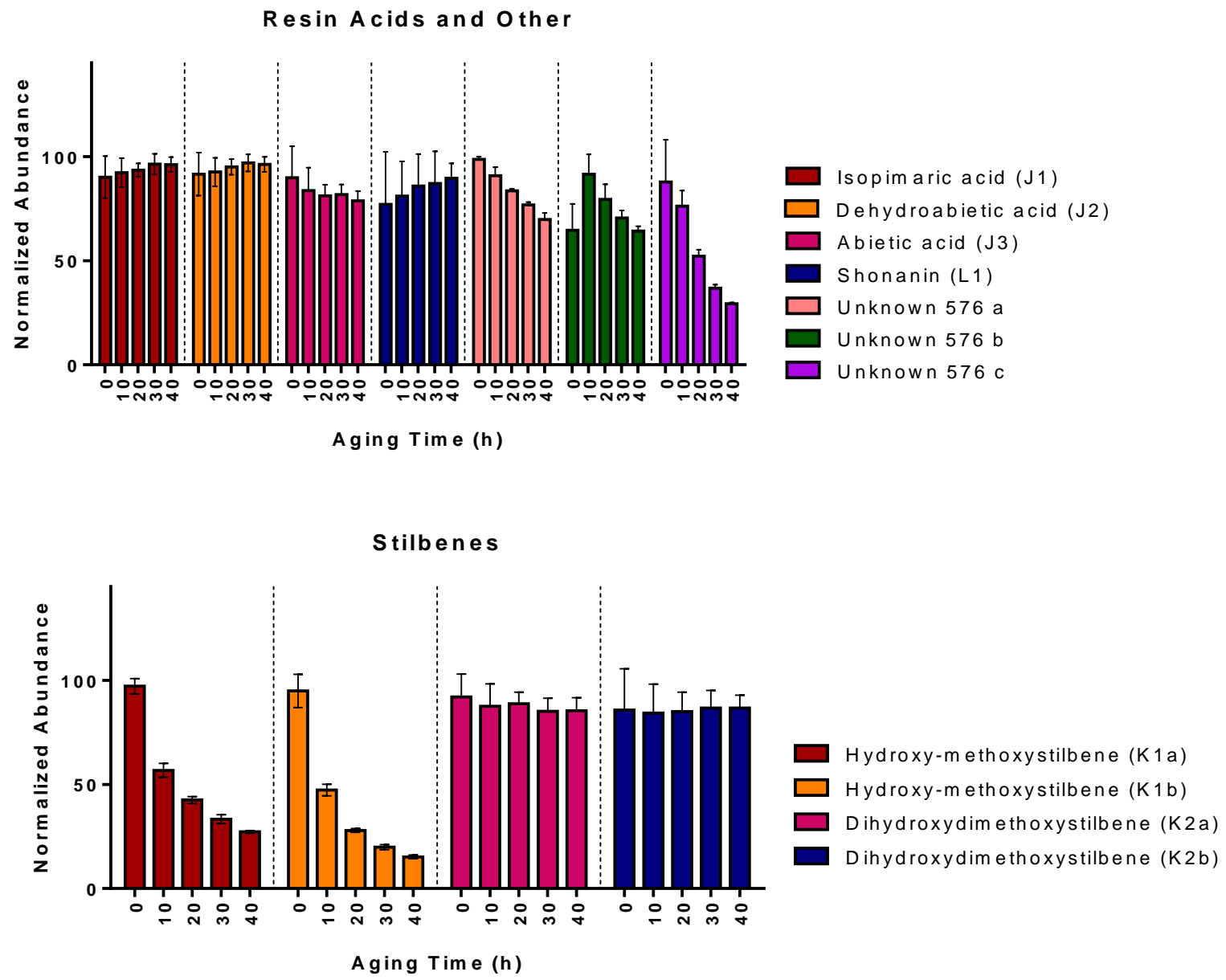

Figure SI-5I. Normalized abundances (mean $\pm \mathrm{SD} ; \mathrm{n}=3$ ) for resin acids, others, and stilbenes as a function of aging time at $80{ }^{\circ} \mathrm{C}$. 
Table SI-1. Gradient conditions used for LC/MS analyses.

\begin{tabular}{|c|c|c|}
\hline Time & ${\text { Mobile Phase } \mathbf{A}^{\mathbf{a}}}$ & Mobile Phase $^{\mathbf{b}}$ \\
\hline Initial & $99.0 \%$ & $1.0 \%$ \\
\hline $1.50 \mathrm{~min}$ & $87.0 \%$ & $13.0 \%$ \\
\hline $7.50 \mathrm{~min}$ & $85.0 \%$ & $15.0 \%$ \\
\hline $12.5 \mathrm{~min}$ & $80.0 \%$ & $20.0 \%$ \\
\hline $27.5 \mathrm{~min}$ & $60.0 \%$ & $40.0 \%$ \\
\hline $37.5 \mathrm{~min}$ & $40.0 \%$ & $60.0 \%$ \\
\hline $45.0 \mathrm{~min}$ & $15.0 \%$ & $85.0 \%$ \\
\hline $52.5 \mathrm{~min}$ & $5.0 \%$ & $95.0 \%$ \\
\hline $65.0 \mathrm{~min}$ & $0.0 \%$ & $100.0 \%$ \\
\hline $65.1 \mathrm{~min}$ & $0.0 \%$ & $100.0 \%$ \\
\hline
\end{tabular}

${ }^{a}$ Mobile phase A: $0.1 \%$ formic acid in water; ${ }^{b}$ Mobile phase B: $0.1 \%$ formic acid and $2 \%$ water in acetonitrile.

Table SI-2. Weight average molecular weight, number average molecular weight, and polydispersity for Oil B following accelerated aging at $80{ }^{\circ} \mathrm{C}$, as measured by gel permeation chromatography

\begin{tabular}{|l|c|c|c|c|c|}
\hline Aging Time (h) & $\mathbf{0}$ & $\mathbf{1 0}$ & $\mathbf{2 0}$ & $\mathbf{3 0}$ & $\mathbf{4 0}$ \\
\hline Weight average molecular weight, $\left.\overline{\boldsymbol{M}}_{\boldsymbol{n}}, \mathbf{( g / m o l}\right)$ & 393.4 & 471.6 & 540.7 & 613.0 & 632.4 \\
\hline Number average molecular weight, $\left.\overline{\boldsymbol{M}}_{\boldsymbol{w}} \mathbf{( g / m o l}\right)$ & 109.2 & 117.8 & 132.7 & 148.9 & 144.6 \\
\hline Polydispersity, $\overline{\boldsymbol{M}}_{\boldsymbol{w}} / \overline{\boldsymbol{M}}_{\boldsymbol{n}}$ & 3.6 & 4.0 & 4.1 & 4.1 & 4.4 \\
\hline
\end{tabular}


Table SI-3. Oil B components detected and quantified (wt $\%$ ) following derivatization with MSFTA and GC/MS analysis, sorted by retention time, for oils before and after $40 \mathrm{~h}$ of aging at $80{ }^{\circ} \mathrm{C}$.

\begin{tabular}{|c|c|c|c|c|c|c|c|c|c|c|c|}
\hline 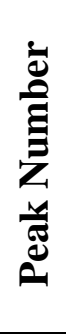 & 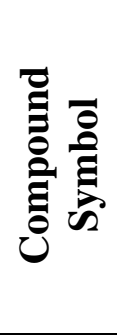 & Compound Name & 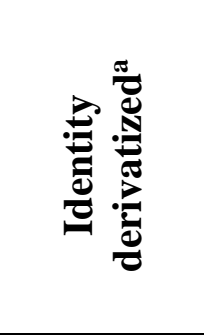 & 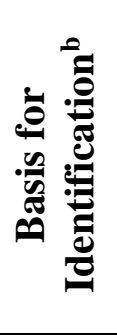 & 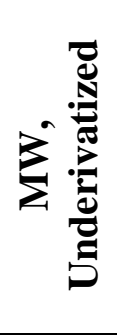 & 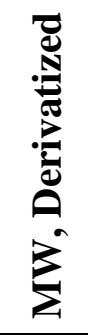 & 疍 & 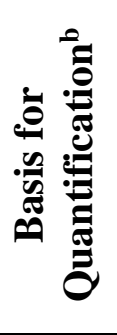 & & 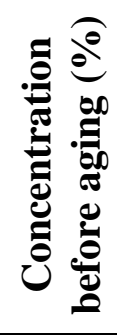 & 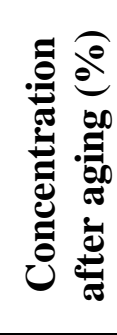 \\
\hline 1 & B1 & 1-Hydroxy-2-propanone & monoTMS & $\mathrm{S}$ & 74 & 146 & 8.08 & $\mathrm{~S}$ & 131 & 0.701 & 0.691 \\
\hline 2 & A1 & Formaldehyde & $\begin{array}{c}\text { MSTFA } \\
\text { adduct }\end{array}$ & $\mathrm{S}$ & 30 & 229 & 13.36 & $\mathrm{~S}^{*}$ & 184 & 0.245 & 0.060 \\
\hline 3 & $\mathrm{~A} 2$ & Acetaldehyde & $\begin{array}{c}\text { MSTFA } \\
\text { adduct }\end{array}$ & $\mathrm{S}$ & 44 & 243 & 13.59 & $\mathrm{~S}$ & 184 & 0.066 & 0.030 \\
\hline 4 & $\mathrm{~B} 2$ & Ethylene glycol & diTMS & $\mathrm{S}$ & 62 & 206 & 13.89 & $\mathrm{~S}$ & 147 & 0.174 & 0.139 \\
\hline 5 & F1 & 2-Furanmethanol & TMS & $S$ & 98 & 170 & 14.37 & $S$ & 155 & 0.060 & 0.010 \\
\hline 6 & $\mathrm{P} 1$ & Phenol & monoTMS & $\mathrm{S}$ & 94 & 166 & 17.42 & $\mathrm{~S}$ & 151 & 0.042 & 0.044 \\
\hline 7 & $\mathrm{C} 1$ & Lactic acid & diTMS & $\mathrm{S}$ & 90 & 234 & 18.22 & $S$ & 147 & 0.007 & 0.010 \\
\hline 8 & $\mathrm{C} 3$ & Hexanoic acid & monoTMS & Lib & 116 & 188 & 18.97 & $\mathrm{~S}$ & TIC & 0.019 & 0.023 \\
\hline 9 & $\mathrm{C} 2$ & Glycolic acid & diTMS & $S$ & 76 & 220 & 19.22 & $S$ & 147 & 0.006 & 0.008 \\
\hline 10 & F5 & Furoic acid & monoTMS & $S$ & 112 & 184 & 20.24 & $\mathrm{~S}$ & 169 & 0.007 & 0.009 \\
\hline 11 & a1 & Unknown aldehyde 1 & $\begin{array}{c}\text { MSTFA } \\
\text { adduct, } \\
\text { nTMS }\end{array}$ & I & --- & --- & 21.60 & $\begin{array}{l}\text { SR- } \\
\text { TIC }\end{array}$ & TIC & 0.037 & 0.002 \\
\hline 12 & $\mathrm{P} 2$ & $o$-Cresol & monoTMS & $S$ & 108 & 180 & 21.73 & $\mathrm{~S}$ & 180 & 0.026 & 0.025 \\
\hline 13 & A3 & 2-Butenal (Crotonaldehyde) & $\begin{array}{c}\text { MSTFA } \\
\text { adduct }\end{array}$ & $\mathrm{S}$ & 70 & 269 & 21.75 & $\mathrm{~S}$ & 143 & 0.424 & 0.199 \\
\hline 14 & $\mathrm{C} 4$ & Levulinic acid & monoTMS & $S$ & 120 & 188 & 22.29 & $S$ & 173 & 0.032 & 0.065 \\
\hline 14 & B3 & $\begin{array}{c}\text { 2-Hydroxy-2-cyclopenten-1- } \\
\text { one }\end{array}$ & monoTMS & $\mathrm{S}$ & 98 & 170 & 22.32 & $\mathrm{~S}$ & 155 & 0.474 & 0.176 \\
\hline
\end{tabular}




\begin{tabular}{|c|c|c|c|c|c|c|c|c|c|c|c|}
\hline 15 & P3 & $m$-Cresol & monoTMS & $\mathrm{S}$ & 108 & 180 & 22.32 & $\mathrm{~S}$ & 180 & 0.021 & 0.021 \\
\hline 16 & $\mathrm{P} 4$ & $p$-Cresol & monoTMS & $\mathrm{S}$ & 108 & 180 & 22.89 & $\mathrm{~S}$ & 180 & 0.027 & 0.025 \\
\hline 17 & $\mathrm{a} 2$ & Unknown aldehyde 2 & $\begin{array}{l}\text { MSFTA } \\
\text { adduct; } \\
\text { nTMS }\end{array}$ & $\mathrm{I}$ & --- & --- & 22.96 & NQ & NQ & NQ & NQ \\
\hline 18 & B4 & $\begin{array}{l}\text { 2-Hydroxy-3-methyl-2- } \\
\text { cyclopenten-1-one }\end{array}$ & monoTMS & $\mathrm{S}$ & 112 & 184 & 25.05 & $\mathrm{~S}^{*}$ & 169 & 0.258 & 0.238 \\
\hline 19 & a3 & Unknown aldehyde 3 & $\begin{array}{l}\text { MSFTA } \\
\text { adduct; } \\
\text { nTMS }\end{array}$ & $\mathrm{I}$ & --- & --- & 25.33 & NQ & NQ & NQ & NQ \\
\hline 20 & B5 & 2-Deoxytetrono-1,4-lactone & monoTMS & $\mathrm{Lit}^{1}$ & 102 & 174 & 25.93 & $\begin{array}{l}\text { SR- } \\
\text { TIC }\end{array}$ & TIC & 0.271 & 0.297 \\
\hline 21 & P5 & 2,4-Dimethylphenol & monoTMS & $\mathrm{S}$ & 122 & 194 & 26.18 & $S$ & 194 & 0.017 & 0.019 \\
\hline 22 & G1 & Guaiacol & monoTMS & $S$ & 124 & 196 & 26.24 & $\mathrm{~S}$ & 136 & 0.340 & 0.341 \\
\hline 23 & B6 & 1,3-Dihydroxy-propan-2-one & diTMS & Lib & 90 & 234 & 26.44 & $\begin{array}{l}\text { SR- } \\
\text { TIC }\end{array}$ & $\mathrm{TIC}$ & 0.043 & 0.020 \\
\hline 24 & A4 & Glycolaldehyde & $\begin{array}{c}\text { monoTMS; } \\
\text { MSFTA } \\
\text { adduct }\end{array}$ & $\mathrm{S}$ & 60 & 331 & 27.27 & $S^{*}$ & TIC & 0.850 & 0.243 \\
\hline 25 & $\mathrm{~F} 2$ & Furfural & $\begin{array}{c}\text { MSFTA } \\
\text { adduct }\end{array}$ & $\mathrm{S}$ & 96 & 295 & 27.35 & $\mathrm{~S}$ & 169 & 0.224 & 0.203 \\
\hline 26 & B7 & Glycerol & triTMS & $S$ & 92 & 308 & 28.56 & $S$ & 205 & 0.059 & 0.012 \\
\hline 27 & B8a & Glycolaldehyde dimer, 1 & diTMS & $\mathrm{S}$ & 120 & 264 & 28.75 & $\begin{array}{l}\text { SR- } \\
\text { EIC }\end{array}$ & 161 & 0.078 & 0.013 \\
\hline 28 & E1 & $\begin{array}{c}\text { 1,2-Dihydroxybenzene (o- } \\
\text { Catechol) }\end{array}$ & diTMS & $\mathrm{S}$ & 110 & 254 & 29.69 & $\mathrm{~S}$ & 254 & 0.300 & 0.296 \\
\hline 29 & $\mathrm{a} 4$ & Unknown aldehyde 4 & $\begin{array}{l}\text { MSFTA } \\
\text { adduct; } \\
\text { nTMS }\end{array}$ & I & --- & --- & 29.76 & NQ & NQ & NQ & NQ \\
\hline 30 & $\mathrm{G} 2$ & 4-Methylguaiacol & monoTMS & $\mathrm{S}$ & 138 & 210 & 29.85 & $\mathrm{~S}$ & 210 & 0.380 & 0.389 \\
\hline 31 & $\mathrm{~B} 8 \mathrm{~b}$ & Glycolaldehyde dimer, 2 & diTMS & $\mathrm{S}$ & 120 & 264 & 29.98 & $\begin{array}{l}\text { SR- } \\
\text { EIC }\end{array}$ & 161 & 0.071 & 0.011 \\
\hline
\end{tabular}




\begin{tabular}{|c|c|c|c|c|c|c|c|c|c|c|c|}
\hline 32 & $\mathrm{~F} 3$ & 5-Methyl furfural & $\begin{array}{c}\text { MSFTA } \\
\text { adduct }\end{array}$ & $\mathrm{S}$ & 110 & 309 & 29.99 & $S$ & 183 & 0.030 & 0.027 \\
\hline 33 & a5 & Unknown aldehyde 5 & $\begin{array}{c}\text { MSFTA } \\
\text { adduct; } \\
\text { nTMS }\end{array}$ & I & --- & --- & 30.64 & NQ & NQ & NQ & NQ \\
\hline 34 & A5 & Hexanal & $\begin{array}{c}\text { MSFTA } \\
\text { adduct }\end{array}$ & $\mathrm{Lit}^{2}$ & 100 & 299 & 30.73 & NQ & NQ & NQ & NQ \\
\hline 35 & IS & Biphenyl (Internal Standard) & ---- & $S$ & --- & --- & 32.01 & --- & --- & --- & --- \\
\hline 36 & $\mathrm{E} 2$ & 1,3-Dihydroxybenzene & diTMS & $\mathrm{S}$ & 110 & 254 & 32.21 & $\mathrm{~S}$ & 254 & 0.002 & 0.000 \\
\hline 37 & D1 & 4-Methylcatechol & monoTMS & $\mathrm{S}$ & 124 & 268 & 32.33 & $S$ & 268 & 0.125 & 0.113 \\
\hline 38 & a6 & Unknown aldehyde 6 & $\begin{array}{c}\text { MSFTA } \\
\text { adduct; } \\
\text { nTMS }\end{array}$ & I & --- & --- & 32.50 & NQ & NQ & NQ & NQ \\
\hline 39 & G3 & 4-Ethylguaiacol & monoTMS & $\mathrm{S}$ & 152 & 224 & 32.53 & $S$ & 224 & 0.082 & 0.080 \\
\hline 40 & D2 & 3-Methylcatechol & diTMS & $\mathrm{S}$ & 124 & 268 & 32.61 & $S$ & 268 & 0.081 & 0.073 \\
\hline 41 & $\mathrm{~S} 1$ & Syringol & monoTMS & $S$ & 154 & 226 & 32.74 & $S$ & 226 & 0.057 & 0.059 \\
\hline 42 & E3 & 1,4-Dihydroxybenzene & diTMS & $\mathrm{S}$ & 110 & 254 & 32.87 & $\mathrm{~S}$ & 254 & 0.089 & 0.089 \\
\hline 43 & h1 & $\begin{array}{l}\text { Unknown monosaccharide, } \\
\text { furanose, h1 }\end{array}$ & nTMS & I & 162 & 378 & 32.92 & $\begin{array}{l}\text { SR- } \\
\text { EIC }\end{array}$ & 217 & 0.141 & 0.006 \\
\hline 44 & G4 & 4-Vinylguaiacol & monoTMS & $S$ & 150 & 222 & 34.00 & $S$ & 222 & 0.104 & 0.013 \\
\hline 45 & D3 & 4-Ethylcatechol & diTMS & $\mathrm{S}$ & 138 & 282 & 34.66 & $\mathrm{~S}$ & 282 & 0.046 & 0.043 \\
\hline 46 & G6 & Eugenol & monoTMS & $\mathrm{S}$ & 164 & 236 & 35.07 & $\mathrm{~S}$ & 236 & 0.135 & 0.139 \\
\hline 47 & G5 & 4-Propylguaiacol & monoTMS & S & 166 & 238 & 35.23 & $\begin{array}{l}\text { SR- } \\
\text { TIC }\end{array}$ & TIC & 0.038 & 0.037 \\
\hline 48 & $\mathrm{~S} 2$ & 4-Methylsyringol & monoTMS & $\mathrm{S}$ & 168 & 240 & 35.41 & $\begin{array}{l}\text { SR- } \\
\text { TIC }\end{array}$ & TIC & 0.060 & 0.056 \\
\hline 49 & a7 & Unknown aldehyde 7 & $\begin{array}{c}\text { MSFTA } \\
\text { adduct; } \\
\text { nTMS }\end{array}$ & I & --- & --- & 35.89 & NQ & NQ & NQ & NQ \\
\hline 50 & G7 & Isoeugenol $(Z)$ & monoTMS & $\mathrm{S}$ & 164 & 236 & 36.62 & $\mathrm{~S}$ & 236 & 0.101 & 0.091 \\
\hline 51 & $\mathrm{E} 4$ & 1,2,3-Trihydroxybenzene & triTMS & $S$ & 126 & 342 & 37.14 & $S$ & 342 & 0.216 & 0.021 \\
\hline
\end{tabular}




\begin{tabular}{|c|c|c|c|c|c|c|c|c|c|c|c|}
\hline 52 & S3 & 4-Ethylsyringol & monoTMS & $\mathrm{I}$ & 182 & 254 & 37.49 & $\begin{array}{l}\text { SR- } \\
\text { EIC }\end{array}$ & 254 & 0.010 & 0.010 \\
\hline 53 & G8 & Isoeugenol $(E)$ & monoTMS & $S$ & 164 & 236 & 38.08 & $\mathrm{~S}$ & 236 & 0.498 & 0.354 \\
\hline 54 & S4 & 4-Vinylsyringol & monoTMS & $\mathrm{Lib}$ & 180 & 252 & 38.79 & $\begin{array}{l}\text { SR- } \\
\text { EIC }\end{array}$ & 222 & 0.071 & 0.008 \\
\hline 55 & a8 & Unknown aldehyde 8 & $\begin{array}{l}\text { MSFTA } \\
\text { adduct; } \\
\text { nTMS }\end{array}$ & I & --- & --- & 38.95 & NQ & NQ & NQ & NQ \\
\hline 56 & E5 & 1,2,4-Trihydroxybenzene & triTMS & $\mathrm{S}$ & 126 & 342 & 39.04 & $S$ & 342 & 0.048 & 0.000 \\
\hline 57 & G10 & Acetovanillone & monoTMS & Lib & 166 & 238 & 39.48 & $\begin{array}{l}\text { SR- } \\
\text { EIC }\end{array}$ & 238 & 0.161 & 0.167 \\
\hline 58 & $\mathrm{H} 4(\mathrm{a}, \mathrm{f})$ & $\begin{array}{c}\text { H4(a,f) alpha } \\
\text { arabinofuranose }\end{array}$ & triTMS & $\mathrm{S}$ & 150 & 438 & 39.53 & $\begin{array}{l}\text { SR- } \\
\text { EIC }\end{array}$ & 217 & 0.011 & 0.124 \\
\hline 59 & $\mathrm{~F} 4$ & 5-Hydroxymethyl furfural & $\begin{array}{c}\text { monoTMS; } \\
\text { MSFTA } \\
\text { adduct }\end{array}$ & $S$ & 126 & 397 & 39.56 & $\mathrm{~S}$ & 108 & 0.338 & 0.291 \\
\hline 60 & $\mathrm{H} 4(\mathrm{a}, \mathrm{p})$ & $\begin{array}{c}\mathrm{H} 4(\mathrm{a}, \mathrm{p}) \text { alpha } \\
\text { arabinopyranose }\end{array}$ & pentaTMS & $\mathrm{S}$ & 150 & 438 & 39.60 & $\begin{array}{l}\text { SR- } \\
\text { EIC }\end{array}$ & 204 & 0.005 & 0.042 \\
\hline 61 & S5 & 4-Ethenylsyringol & monoTMS & I & 194 & 266 & 39.64 & $\begin{array}{l}\text { SR- } \\
\text { EIC }\end{array}$ & 266 & 0.016 & 0.017 \\
\hline 62 & a9 & Unknown aldehyde 9 & $\begin{array}{l}\text { MSFTA } \\
\text { adduct; } \\
\text { nTMS }\end{array}$ & $\mathrm{I}$ & --- & --- & 39.85 & NQ & NQ & NQ & NQ \\
\hline 63 & $\mathrm{H} 5(\mathrm{a}, \mathrm{f})$ & H5(a,f) alpha xylofuranose & nTMS & $S$ & 150 & 438 & 39.90 & $\begin{array}{l}\text { SR- } \\
\text { EIC }\end{array}$ & 217 & 0.043 & 0.085 \\
\hline 64 & G11 & Guaiacylacetone & monoTMS & $\mathrm{Lib}$ & 180 & 252 & 40.05 & $\begin{array}{l}\text { SR- } \\
\text { TIC }\end{array}$ & TIC & 0.148 & 0.153 \\
\hline 65 & $\mathrm{H} 5(\mathrm{~b}, \mathrm{f})$ & H5(b,f) beta xylofuranose & nTMS & S & 150 & 438 & 40.27 & $\begin{array}{l}\text { SR- } \\
\text { EIC }\end{array}$ & 217 & 0.020 & 0.087 \\
\hline 66 & $\mathrm{H} 4(\mathrm{~b}, \mathrm{p})$ & $\begin{array}{c}\mathrm{H} 4(\mathrm{~b}, \mathrm{p}) \text { beta } \\
\text { arabinopyranose }\end{array}$ & pentaTMS & $\mathrm{S}$ & 150 & 438 & 40.43 & $\begin{array}{l}\text { SR- } \\
\text { EIC }\end{array}$ & 204 & 0.004 & 0.063 \\
\hline
\end{tabular}




\begin{tabular}{|c|c|c|c|c|c|c|c|c|c|c|c|}
\hline 67 & a10 & Unknown aldehyde 10 & $\begin{array}{c}\text { MSFTA } \\
\text { adduct; } \\
\text { nTMS }\end{array}$ & I & --- & --- & 40.53 & NQ & NQ & NQ & NQ \\
\hline 68 & a11 & Unknown aldehyde 11 & $\begin{array}{c}\text { MSFTA } \\
\text { adduct; } \\
\text { nTMS }\end{array}$ & $\mathrm{I}$ & --- & --- & 40.72 & NQ & NQ & NQ & NQ \\
\hline 69 & $\mathrm{H} 1$ & Galactosan & triTMS & $\mathrm{Lit}^{3,4}$ & 162 & 378 & 40.78 & $\begin{array}{l}\text { SR- } \\
\text { EIC }\end{array}$ & 217 & 0.254 & 0.203 \\
\hline 70 & S6 & $\begin{array}{l}\text { 4-(1-Propenyl)-2,6- } \\
\text { dimethoxyphenol ( } Z\end{array}$ & monoTMS & I & 194 & 266 & 40.99 & $\begin{array}{l}\text { SR- } \\
\text { EIC }\end{array}$ & 266 & 0.013 & 0.011 \\
\hline 71 & $\mathrm{H} 4(\mathrm{~b}, \mathrm{f})$ & $\mathrm{H} 4(\mathrm{~b}, \mathrm{f})$ beta arabinofuranose & nTMS & $\mathrm{S}$ & 150 & 438 & 41.24 & $\begin{array}{l}\text { SR- } \\
\text { EIC }\end{array}$ & 217 & 0.016 & 0.091 \\
\hline 72 & $\mathrm{H} 2$ & Mannosan & triTMS & $\mathrm{Lit}^{3,4}$ & 162 & 378 & 41.35 & $\begin{array}{l}\text { SR- } \\
\text { EIC }\end{array}$ & 333 & 1.226 & 1.011 \\
\hline 73 & $\mathrm{~h} 2$ & $\begin{array}{l}\text { Unknown monosaccharide, } \\
\text { furanose, h2 }\end{array}$ & nTMS & --- & 162 & 378 & 41.68 & $\begin{array}{l}\text { SR- } \\
\text { EIC }\end{array}$ & 217 & 0.070 & 0.065 \\
\hline 74 & $\mathrm{H} 3$ & Levoglucosan & triTMS & $S$ & 162 & 378 & 41.99 & $\mathrm{~S}$ & 333 & 3.852 & 3.847 \\
\hline 75 & G12 & Homovanillyl alcohol & diTMS & Lib & 168 & 312 & 42.04 & $\begin{array}{l}\text { SR- } \\
\text { EIC }\end{array}$ & 312 & 0.057 & 0.028 \\
\hline 76 & $\mathrm{H} 5(\mathrm{a}, \mathrm{p})$ & H5(a,p) alpha xylopyranose & pentaTMS & S & 150 & 438 & 42.22 & $\begin{array}{l}\text { SR- } \\
\text { EIC }\end{array}$ & 204 & 0.029 & 0.142 \\
\hline 77 & a12 & Unknown aldehyde 12 & $\begin{array}{c}\text { MSFTA } \\
\text { adduct; } \\
\text { nTMS }\end{array}$ & $\mathrm{I}$ & --- & --- & 42.44 & NQ & NQ & NQ & NQ \\
\hline 78 & h3 & $\begin{array}{l}\text { Unknown monosaccharide, } \\
\text { furanose, } \mathrm{h} 3\end{array}$ & nTMS & --- & 162 & 378 & 42.44 & $\begin{array}{l}\text { SR- } \\
\text { EIC }\end{array}$ & 217 & 0.268 & 0.037 \\
\hline 79 & S7 & $\begin{array}{l}\text { 4-(1-Propenyl)-2,6- } \\
\text { dimethoxyphenol }(E)\end{array}$ & monoTMS & I & 194 & 266 & 42.57 & $\begin{array}{l}\text { SR- } \\
\text { EIC }\end{array}$ & 266 & 0.059 & 0.046 \\
\hline 80 & $\mathrm{~h} 4$ & $\begin{array}{l}\text { Unknown monosaccharide, } \\
\text { furanose, h4 }\end{array}$ & triTMS & --- & --- & --- & 42.92 & $\begin{array}{l}\text { SR- } \\
\text { EIC }\end{array}$ & 217 & 0.101 & 0.101 \\
\hline 81 & h5 & $\begin{array}{l}\text { Unknown monosaccharide, } \\
\text { furanose, } \mathrm{h} 5\end{array}$ & nTMS & --- & --- & --- & 43.22 & $\begin{array}{l}\text { SR- } \\
\text { EIC }\end{array}$ & 217 & 0.074 & 0.000 \\
\hline
\end{tabular}




\begin{tabular}{|c|c|c|c|c|c|c|c|c|c|c|c|}
\hline 82 & h6 & $\begin{array}{l}\text { Unknown monosaccharide, } \\
\text { furanose, h6 }\end{array}$ & nTMS & --- & --- & --- & 43.33 & $\begin{array}{l}\text { SR- } \\
\text { EIC }\end{array}$ & 217 & 0.162 & 0.003 \\
\hline 83 & G13 & Vanillic Acid & $\operatorname{diTMS}$ & & 168 & 312 & 43.56 & $\begin{array}{l}\text { SR- } \\
\text { EIC }\end{array}$ & 312 & 0.066 & 0.072 \\
\hline 84 & $\mathrm{H} 5(\mathrm{~b}, \mathrm{p})$ & H5(b,p) beta xylopyranose & pentaTMS & $\mathrm{S}$ & 150 & 438 & 43.62 & $\begin{array}{l}\text { SR- } \\
\text { EIC }\end{array}$ & 204 & 0.026 & 0.152 \\
\hline 85 & h7 & $\begin{array}{l}\text { Unknown monosaccharide, } \\
\text { furanose, } \mathrm{h} 7\end{array}$ & nTMS & --- & 324 & 756 & 43.93 & $\begin{array}{l}\text { SR- } \\
\text { EIC }\end{array}$ & 217 & 0.205 & 0.011 \\
\hline 86 & a13 & Unknown aldehyde 13 & $\begin{array}{l}\text { MSFTA } \\
\text { adduct; } \\
\text { nTMS }\end{array}$ & $\mathrm{I}$ & --- & --- & 44.21 & $\begin{array}{l}\text { SR- } \\
\text { TIC }\end{array}$ & TIC & 0.060 & 0.050 \\
\hline 87 & G9 & Vanillin & monoTMS & $\mathrm{S}$ & 152 & 423 & 44.81 & $\mathrm{~S}$ & 297 & 0.238 & 0.229 \\
\hline 88 & G14 & 3-Vanilpropanol & $\operatorname{diTMS}$ & Lib & 182 & 326 & 44.93 & $\begin{array}{l}\text { SR- } \\
\text { TIC }\end{array}$ & TIC & 0.432 & 0.335 \\
\hline 89 & B9 & Pinitol & pentaTMS & $\mathrm{Lit}^{5}$ & 194 & 554 & 45.28 & $\begin{array}{l}\text { SR- } \\
\text { EIC }\end{array}$ & 260 & 0.296 & 0.336 \\
\hline 90 & G15 & Coniferyl alcohol $(Z)$ & diTMS & $\mathrm{S}$ & 180 & 324 & 45.28 & $\begin{array}{l}\text { SR- } \\
324\end{array}$ & 324 & 0.401 & 0.045 \\
\hline 91 & h8 & $\begin{array}{l}\text { Unknown monosaccharide, } \\
\text { furanose }, \mathrm{h} 8\end{array}$ & nTMS & --- & 342 & 918 & 45.83 & $\begin{array}{l}\text { SR- } \\
\text { EIC }\end{array}$ & 217 & 0.168 & 0.167 \\
\hline 92 & a14 & Unknown aldehyde 14 & $\begin{array}{l}\text { MSFTA } \\
\text { adduct; } \\
\text { nTMS }\end{array}$ & $\mathrm{I}$ & --- & --- & 46.12 & $\begin{array}{l}\text { SR- } \\
\text { TIC }\end{array}$ & TIC & 0.168 & 0.130 \\
\hline 93 & H6(a,p) & H6(a,p) alpha-glucopyranose & pentaTMS & $\mathrm{S}$ & 180 & 540 & 46.89 & $\begin{array}{l}\text { SR- } \\
\text { EIC }\end{array}$ & 204 & 0.033 & 0.117 \\
\hline 94 & S8 & Syringaldehyde & $\begin{array}{c}\text { monoTMS; } \\
\text { MSTFA } \\
\text { adduct }\end{array}$ & $\mathrm{Lib}$ & 182 & 453 & 47.28 & $\begin{array}{l}\text { SR- } \\
\text { EIC }\end{array}$ & 327 & 0.036 & 0.036 \\
\hline 95 & a15 & Unknown aldehyde 15 & $\begin{array}{l}\text { MSFTA } \\
\text { adduct; } \\
\text { nTMS }\end{array}$ & $\mathrm{I}$ & --- & --- & 47.59 & NQ & NQ & NQ & NQ \\
\hline 96 & G16 & Coniferyl alcohol $(E)$ & $\operatorname{diTMS}$ & $S$ & 180 & 324 & 48.02 & $\begin{array}{l}\text { SR- } \\
324\end{array}$ & 324 & 0.215 & 0.000 \\
\hline
\end{tabular}




\begin{tabular}{|c|c|c|c|c|c|c|c|c|c|c|c|}
\hline 97 & h9 & Unknown disaccharide, h9 & nTMS & --- & --- & --- & 48.62 & $\begin{array}{l}\text { SR- } \\
\text { EIC }\end{array}$ & 217 & 0.080 & 0.065 \\
\hline 98 & a16 & Unknown aldehyde 16 & $\begin{array}{l}\text { MSFTA } \\
\text { adduct; } \\
\text { nTMS }\end{array}$ & I & --- & --- & 49.12 & NQ & NQ & NQ & NQ \\
\hline 99 & H6(b,p) & H6(b,p) beta-glucopyranose & pentaTMS & S & 180 & 540 & 49.19 & $\begin{array}{l}\text { SR- } \\
\text { EIC }\end{array}$ & 204 & 0.019 & 0.110 \\
\hline 100 & K1a & $\begin{array}{l}\text { Methoxy/hydroxy stilbene } \\
\text { (Z) }\end{array}$ & monoTMS & I & 226 & 298 & 50.45 & $\begin{array}{l}\text { SR- } \\
\text { EIC }\end{array}$ & 298 & 0.010 & 0.002 \\
\hline 101 & $\mathrm{C} 4$ & Palmitic acid & monoTMS & $S$ & 116 & 188 & 50.60 & SR- & 313 & 0.130 & 0.129 \\
\hline 102 & a17 & Unknown aldehyde 17 & $\begin{array}{l}\text { MSFTA } \\
\text { adduct; } \\
\text { nTMS }\end{array}$ & I & --- & --- & 50.83 & NQ & NQ & NQ & NQ \\
\hline 103 & h10 & Unknown disaccharide, h10 & nTMS & I & --- & --- & 51.21 & $\begin{array}{l}\text { SR- } \\
\text { EIC }\end{array}$ & 217 & 0.143 & 0.132 \\
\hline 104 & G17 & Coniferylaldehyde $(E)$ & $\begin{array}{c}\text { monoTMS; } \\
\text { MSTFA } \\
\text { adduct }\end{array}$ & S & 178 & 250 & 53.24 & $\begin{array}{l}\text { SR- } \\
449\end{array}$ & 449 & 0.370 & 0.261 \\
\hline 105 & h11 & Unknown disaccharide, h11 & nTMS & I & --- & --- & 53.58 & $\begin{array}{l}\text { SR- } \\
\text { EIC }\end{array}$ & 275 & 0.096 & 0.069 \\
\hline 106 & $\mathrm{H} 7$ & H7, Cellobiosan & hexaTMS & S & 324 & 756 & 53.92 & $\begin{array}{l}\text { SR- } \\
\text { EIC }\end{array}$ & 204 & 0.226 & 0.225 \\
\hline 107 & C5 & Oleic acid & monoTMS & S & 256 & 328 & 54.36 & $\begin{array}{l}\text { SR- } \\
\text { EIC }\end{array}$ & 339 & 0.319 & 0.309 \\
\hline 108 & $\mathrm{~K} 1 \mathrm{~b}$ & $\begin{array}{l}\text { Methoxy/hydroxy stilbene } \\
\text { (E) }\end{array}$ & monoTMS & I & 226 & 298 & 55.94 & $\begin{array}{l}\text { SR- } \\
\text { EIC }\end{array}$ & 298 & 0.046 & 0.006 \\
\hline 109 & S9 & Sinapaldehyde & $\begin{array}{c}\text { monoTMS; } \\
\text { MSTFA } \\
\text { adduct }\end{array}$ & I & 208 & 479 & 55.95 & $\begin{array}{l}\text { SR- } \\
\text { EIC }\end{array}$ & 479 & 0.011 & 0.01 \\
\hline 110 & $\mathrm{~J} 1$ & Isopimaric acid & monoTMS & Lib & 302 & 374 & 56.69 & $\begin{array}{l}\text { SR- } \\
\text { TIC }\end{array}$ & TIC & 0.352 & 0.361 \\
\hline 111 & $\mathrm{~J} 2$ & Dehydroabeitic acid & monoTMS & Lib & 300 & 372 & 57.71 & $\begin{array}{l}\text { SR- } \\
\text { TIC }\end{array}$ & TIC & 0.818 & 0.796 \\
\hline
\end{tabular}




\begin{tabular}{|c|c|c|c|c|c|c|c|c|c|c|c|}
\hline 112 & $\mathrm{~J} 3$ & Abeitic acid & monoTMS & S & 302 & 374 & 58.37 & S-TIC & TIC & 0.193 & 0.153 \\
\hline 113 & $\mathrm{~K} 2 \mathrm{a}$ & $\begin{array}{c}\text { Dimethoxy-dihydroxy } \\
\text { stilbene }(Z)\end{array}$ & diTMS & $\mathrm{I}$ & 272 & 416 & 59.08 & $\begin{array}{l}\text { SR- } \\
\text { TIC }\end{array}$ & TIC & 0.055 & 0.050 \\
\hline 114 & h12 & Unknown disaccharide, h12 & nTMS & --- & --- & --- & 63.26 & $\begin{array}{l}\text { SR- } \\
\text { EIC }\end{array}$ & 361 & 0.338 & 0.153 \\
\hline 115 & $\mathrm{H} 8$ & H8, Cellobiose & nonaTMS & $\mathrm{S}$ & 342 & 918 & 63.71 & $\begin{array}{l}\text { SR- } \\
\text { EIC }\end{array}$ & 361 & 0.884 & 1.209 \\
\hline 116 & h13 & Unknown disaccharide, h13 & nTMS & --- & --- & --- & 63.97 & $\begin{array}{l}\text { SR- } \\
\text { EIC }\end{array}$ & 361 & 0.395 & 0.459 \\
\hline 117 & h14 & Unknown disaccharide, h14 & nTMS & --- & --- & --- & 64.56 & $\begin{array}{l}\text { SR- } \\
\text { EIC }\end{array}$ & 361 & 0.289 & 0.279 \\
\hline 118 & $\mathrm{~K} 2 \mathrm{~b}$ & $\begin{array}{c}\text { Dimethoxy-dihydroxy } \\
\text { stilbene }(\mathrm{E})\end{array}$ & $\operatorname{diTMS}$ & I & 272 & 416 & 65.33 & $\begin{array}{l}\text { SR- } \\
\text { TIC }\end{array}$ & TIC & 0.278 & 0.135 \\
\hline 119 & 11 & Unknown MW = 576 & nTMS & --- & --- & --- & 65.82 & $\begin{array}{l}\text { SR- } \\
\text { TIC }\end{array}$ & TIC & 0.036 & 0.031 \\
\hline 120 & 12 & Unknown $\mathrm{MW}=576$ & nTMS & --- & --- & --- & 65.94 & $\begin{array}{l}\text { SR- } \\
\text { TIC }\end{array}$ & TIC & 0.022 & 0.009 \\
\hline 121 & L1 & Shonanin & diTMS & $\mathrm{Lit}^{6}$ & 344 & 488 & 66.84 & $\begin{array}{l}\text { SR- } \\
\text { TIC }\end{array}$ & TIC & 0.196 & 0.177 \\
\hline 122 & 13 & Unknown MW = 518 & nTMS & --- & --- & --- & 68.73 & $\begin{array}{l}\text { SR- } \\
\text { TIC }\end{array}$ & TIC & 0.055 & 0.066 \\
\hline \multicolumn{10}{|r|}{ Total } & 21.10 & 17.62 \\
\hline
\end{tabular}

${ }^{\mathrm{a}}$ Number of TMS groups added upon derivatization; for aldehyde, a further, net, addition of MSTFA is observed; ' $\mathrm{b}$ Symbols defined on pg SI-3 under "Experimental Details for Quantitative Measurements".

\section{References:}

1. Thompson, J. A.; Markey, S. P.; Fennessey, P. V., Gas-chromatographic/mass-spectrometric identification and quantitation of tetronic and deoxytetronic acids in urine from normal adults and neonates. Clin. Chem. (Winston-Salem, N. C.) 1975, 21 (13), 1892-8.

2. Spiteller, G.; Kern, W.; Spiteller, P., Investigation of aldehydic lipid peroxidation products by gas chromatography-mass spectrometry. Journal of Chromatography A 1999, 843, 29-98.

3. Fabbri, D.; Chiavari, G.; Prati, S.; Vassura, I.; Vangelista, M., Gas chromatography/mass spectrometric characterisation of pyrolysis/silylation products of glucose and cellulose. Rapid Commun. Mass Spectrom. 2002, 16 (24), 2349-2355. 
4. Medeiros, P. M.; Simoneit, B. R. T., Analysis of sugars in environmental samples by gas chromatography-mass spectrometry. J. Chromatogr. A 2007, 1141 (2), 271-278.

5. Garland, S.; Goheen, S.; Donald, P.; McDonald, L.; Campbell, J., Application of derivatization gas chromatography/mass spectrometry for the identification and quantitation of pinitol in plant roots. Anal. Lett. 2009, 42 (13), 2096-2105.

6. Yamamoto, S.; Otto, A.; Simoneit, B. R. T., Lignans in resin of araucaria angustifolia by gas chromatography/mass spectrometry. J. Mass Spectrom. 2004, 39 (11), 1337-1347. 
Table SI-4A. Concentrations of aliphatic aldehydes in Oil B before and after $40 \mathrm{~h}$ of aging at 80 ${ }^{\circ} \mathrm{C}$.

\begin{tabular}{|c|c|c|c|c|c|}
\hline 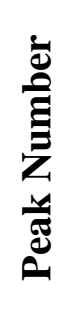 & 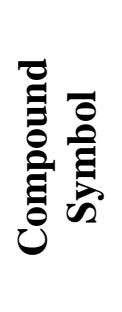 & Compound Name & 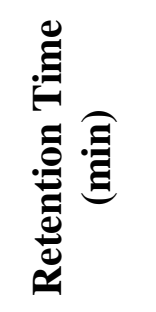 & 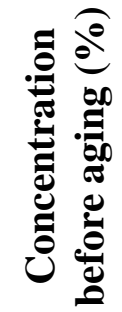 & 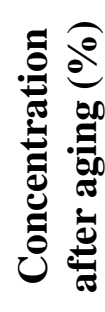 \\
\hline 2 & A1 & Formaldehyde & 13.36 & 0.245 & 0.060 \\
\hline 3 & A2 & Acetaldehyde & 13.59 & 0.066 & 0.030 \\
\hline 13 & A3 & 2-Butenal (Crotonaldehyde) & 21.75 & 0.424 & 0.199 \\
\hline 25 & A4 & Glycolaldehyde & 27.27 & 0.850 & 0.243 \\
\hline 35 & A5 & Hexanal & 30.73 & NQ & $\mathrm{NQ}$ \\
\hline 11 & a1 & Unknown aldehyde 1 & 21.60 & 0.037 & 0.002 \\
\hline 18 & a2 & Unknown aldehyde 2 & 22.96 & NQ & NQ \\
\hline 20 & a3 & Unknown aldehyde 3 & 25.33 & NQ & NQ \\
\hline 30 & a4 & Unknown aldehyde 4 & 29.76 & NQ & NQ \\
\hline 34 & a5 & Unknown aldehyde 5 & 30.64 & NQ & $\mathrm{NQ}$ \\
\hline 39 & a6 & Unknown aldehyde 6 & 32.50 & NQ & $\mathrm{NQ}$ \\
\hline 50 & a7 & Unknown aldehyde 7 & 35.89 & NQ & $\mathrm{NQ}$ \\
\hline 56 & a8 & Unknown aldehyde 8 & 38.95 & NQ & NQ \\
\hline 63 & a9 & Unknown aldehyde 9 & 39.85 & NQ & $\mathrm{NQ}$ \\
\hline 68 & a10 & Unknown aldehyde 10 & 40.53 & NQ & NQ \\
\hline 69 & a11 & Unknown aldehyde 11 & 40.72 & NQ & $\mathrm{NQ}$ \\
\hline 78 & a12 & Unknown aldehyde 12 & 42.44 & NQ & $\mathrm{NQ}$ \\
\hline 87 & a13 & Unknown aldehyde 13 & 44.21 & 0.060 & 0.050 \\
\hline 93 & a14 & Unknown aldehyde 14 & 46.12 & 0.168 & 0.130 \\
\hline 96 & a15 & Unknown aldehyde 15 & 47.59 & NQ & $\mathrm{NQ}$ \\
\hline 99 & a16 & Unknown aldehyde 16 & 49.12 & NQ & $\mathrm{NQ}$ \\
\hline 103 & a17 & Unknown aldehyde 17 & 50.83 & NQ & $\mathrm{NQ}$ \\
\hline \multicolumn{4}{|c|}{ (n) } & 1.849 & 0.714 \\
\hline
\end{tabular}


Table SI-4B. Concentrations of hydroxy ketones and polyols in Oil B before and after $40 \mathrm{~h}$ of aging at $80^{\circ} \mathrm{C}$.

\begin{tabular}{|c|c|c|c|c|c|}
\hline 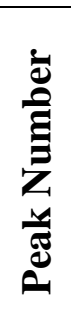 & 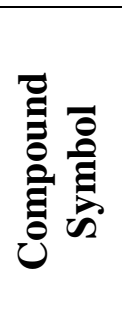 & Compound Name & 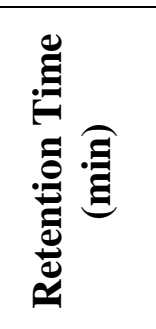 & 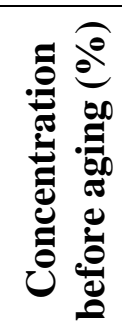 & 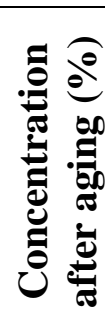 \\
\hline 1 & B1 & 1-Hydroxy-2-propanone & 8.08 & 0.701 & 0.691 \\
\hline 4 & B2 & Ethylene glycol & 13.89 & 0.174 & 0.139 \\
\hline 15 & B3 & 2-Hydroxy-2-cyclopenten-1-one & 22.32 & 0.474 & 0.176 \\
\hline 19 & B4 & $\begin{array}{l}\text { 2-Hydroxy-3-methyl-2-cyclopenten-1- } \\
\text { one }\end{array}$ & 25.05 & 0.258 & 0.238 \\
\hline 21 & B5 & 2-Deoxytetrono-1,4-lactone & 25.93 & 0.271 & 0.297 \\
\hline 24 & B6 & 1,3-Dihydroxy-propan-2-one & 26.44 & 0.043 & 0.020 \\
\hline 27 & B7 & Glycerol & 28.56 & 0.059 & 0.012 \\
\hline 28 & $\mathrm{~B} 8 \mathrm{a}$ & Glycolaldehyde dimer, 1 & 28.75 & 0.078 & 0.013 \\
\hline 32 & $\mathrm{~B} 8 \mathrm{~b}$ & Glycolaldehyde dimer, 2 & 29.98 & 0.071 & 0.011 \\
\hline 90 & B9 & Pinitol & 45.28 & 0.296 & 0.336 \\
\hline & & & TOTAL & 2.425 & 1.933 \\
\hline
\end{tabular}

Table SI-4C. Concentrations of aliphatic acids and hydroxyl acids in Oil B before and after $40 \mathrm{~h}$ of aging at $80^{\circ} \mathrm{C}$.

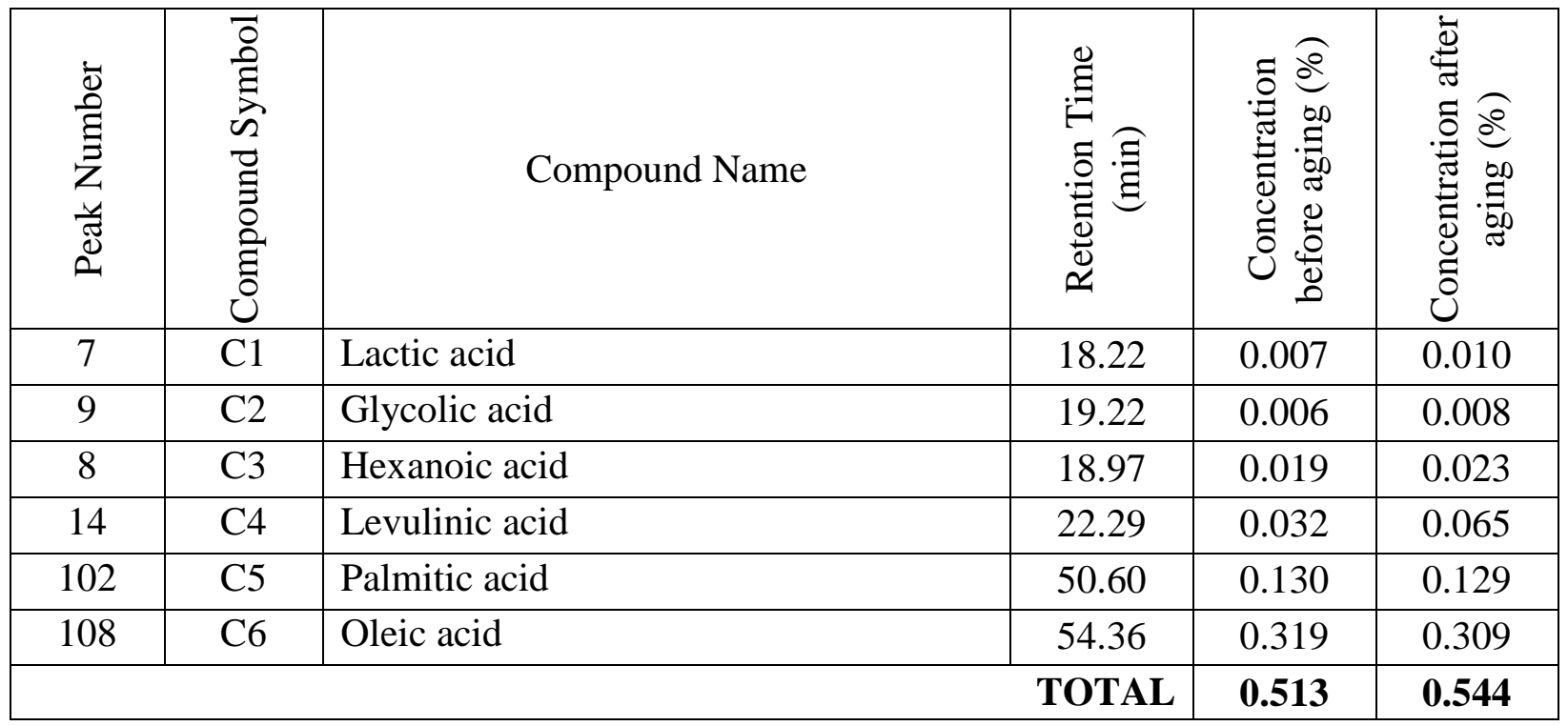


Table SI-4D. Concentrations of phenols in Oil B before and after $40 \mathrm{~h}$ of aging at $80{ }^{\circ} \mathrm{C}$.

\begin{tabular}{|c|c|c|c|c|c|}
\hline 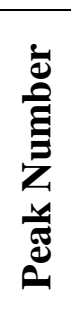 & 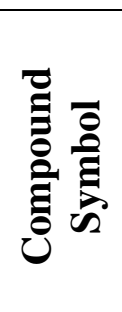 & Compound Name & 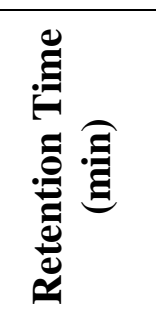 & 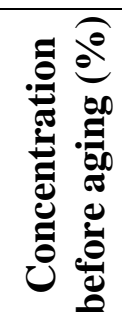 & 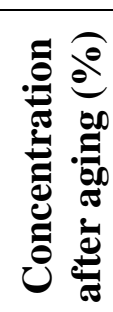 \\
\hline 6 & $\mathrm{P} 1$ & Phenol & 17.42 & 0.042 & 0.044 \\
\hline 12 & $\mathrm{P} 2$ & $o$-Cresol & 21.73 & 0.026 & 0.025 \\
\hline 16 & P3 & $m$-Cresol & 22.32 & 0.021 & 0.021 \\
\hline 17 & $\mathrm{P} 4$ & $p$-Cresol & 22.89 & 0.027 & 0.025 \\
\hline 22 & P5 & 2,4-Dimethylphenol & 26.18 & 0.017 & 0.019 \\
\hline \multicolumn{4}{|r|}{ TOTAL } & 0.133 & 0.134 \\
\hline
\end{tabular}

Table SI-4E. Concentrations of alkyl-substituted catechols in Oil B before and after $40 \mathrm{~h}$ of aging at $80^{\circ} \mathrm{C}$.

\begin{tabular}{|c|c|c|c|c|c|}
\hline 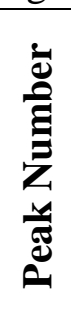 & 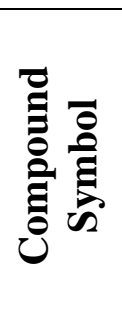 & Compound Name & 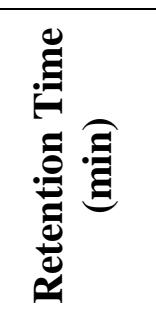 & 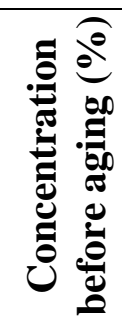 & 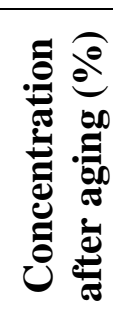 \\
\hline 38 & D1 & 4-Methylcatechol & 32.33 & 0.125 & 0.113 \\
\hline 41 & $\mathrm{D} 2$ & 3-Methylcatechol & 32.61 & 0.081 & 0.073 \\
\hline 46 & D3 & 4-Ethylcatechol & 34.66 & 0.046 & 0.043 \\
\hline \multicolumn{4}{|r|}{ TOTAL } & 0.252 & 0.229 \\
\hline
\end{tabular}


Table SI-4F. Concentrations of hydroxybenzenes in Oil B before and after $40 \mathrm{~h}$ of aging at 80 ${ }^{\circ} \mathrm{C}$.

\begin{tabular}{|c|c|c|c|c|c|}
\hline 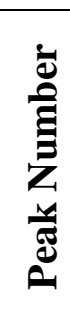 & 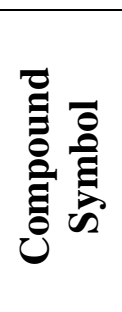 & Compound Name & 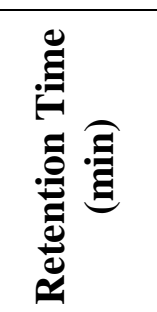 & 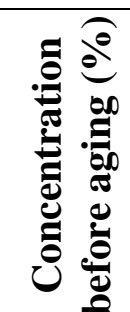 & 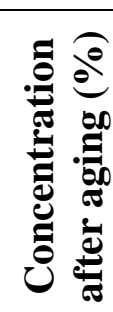 \\
\hline 29 & E1 & 1,2-Dihydroxybenzene (o-Catechol) & 29.69 & 0.300 & 0.296 \\
\hline 37 & E2 & 1,3-Dihydroxybenzene & 32.21 & 0.002 & 0.000 \\
\hline 43 & E3 & 1,4-Dihydroxybenzene & 32.87 & 0.089 & 0.089 \\
\hline 52 & $\mathrm{E} 4$ & 1,2,3-Trihydroxybenzene & 37.14 & 0.216 & 0.021 \\
\hline 57 & E5 & 1,2,4-Trihydroxybenzene & 39.04 & 0.048 & 0.000 \\
\hline \multicolumn{4}{|r|}{ TOTAL } & $\mathbf{0 . 3 5 5}$ & 0.110 \\
\hline
\end{tabular}

Table SI-4G. Concentrations of furans in Oil B before and after $40 \mathrm{~h}$ of aging at $80{ }^{\circ} \mathrm{C}$.

\begin{tabular}{|c|c|c|c|c|c|}
\hline 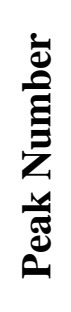 & 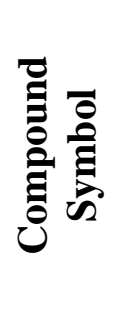 & Compound Name & 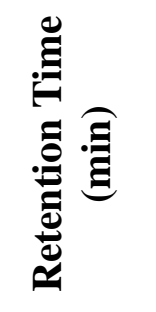 & 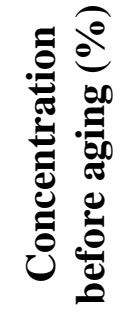 & 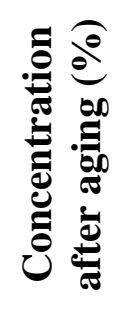 \\
\hline 5 & F1 & 2-Furanmethanol & 14.37 & 0.060 & 0.010 \\
\hline 26 & F2 & Furfural & 27.35 & 0.224 & 0.203 \\
\hline 33 & F3 & 5-Methyl furfural & 29.99 & 0.030 & 0.027 \\
\hline 60 & F4 & 5-Hydroxymethyl furfural & 39.56 & 0.338 & 0.291 \\
\hline 10 & F5 & Furoic acid & 20.24 & 0.007 & 0.009 \\
\hline \multicolumn{4}{|r|}{ TOTAL } & 0.658 & 0.539 \\
\hline
\end{tabular}


Table SI-4H. Concentrations of guaiacols in Oil B before and after $40 \mathrm{~h}$ of aging at $80^{\circ} \mathrm{C}$.

\begin{tabular}{|c|c|c|c|c|c|}
\hline 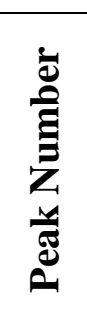 & 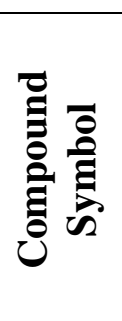 & Compound Name & 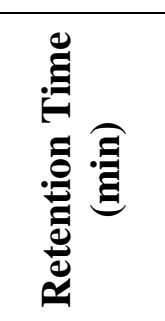 & 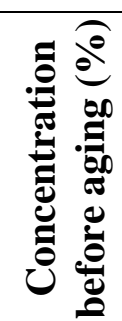 & 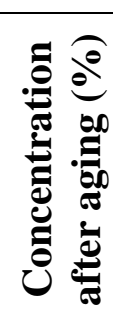 \\
\hline 23 & G1 & Guaiacol & 26.24 & 0.340 & 0.341 \\
\hline 31 & G2 & 4-Methylguaiacol & 29.85 & 0.380 & 0.389 \\
\hline 40 & G3 & 4-Ethylguaiacol & 32.53 & 0.082 & 0.080 \\
\hline 45 & G4 & 4-Vinylguaiacol & 34.00 & 0.104 & 0.013 \\
\hline 48 & G5 & 4-Propylguaiacol & 35.23 & 0.038 & 0.037 \\
\hline 47 & G6 & Eugenol & 35.07 & 0.135 & 0.139 \\
\hline 51 & G7 & Isoeugenol $(Z)$ & 36.62 & 0.101 & 0.091 \\
\hline 54 & G8 & Isoeugenol $(E)$ & 38.08 & 0.498 & 0.354 \\
\hline 88 & G9 & Vanillin & 44.81 & 0.238 & 0.229 \\
\hline 58 & G10 & Acetovanillone & 39.48 & 0.161 & 0.167 \\
\hline 65 & G11 & Guaiacylacetone & 40.05 & 0.148 & 0.153 \\
\hline 76 & G12 & Homovanillyl alcohol & 42.04 & 0.057 & 0.028 \\
\hline 84 & G13 & Vanillic Acid & 43.56 & 0.066 & 0.072 \\
\hline 89 & G14 & 3-Vanilpropanol & 44.93 & 0.432 & 0.335 \\
\hline 91 & G15 & Coniferyl alcohol (Z) & 45.28 & 0.401 & 0.045 \\
\hline 97 & G16 & Coniferyl alcohol $(E)$ & 48.02 & 0.215 & 0.000 \\
\hline 105 & G17 & Coniferylaldehyde $(E)$ & 53.24 & 0.370 & 0.261 \\
\hline \multicolumn{4}{|c|}{ 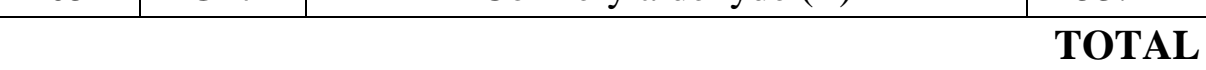 } & 3.765 & 2.735 \\
\hline
\end{tabular}


Table SI-4I. Concentrations of syringols in Oil B before and after $40 \mathrm{~h}$ of aging at $80^{\circ} \mathrm{C}$.

\begin{tabular}{|c|c|c|c|c|c|}
\hline 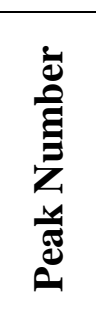 & 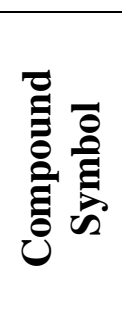 & Compound Name & & 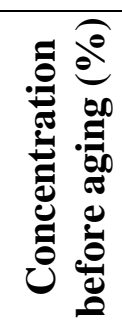 & 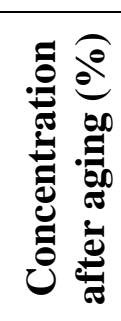 \\
\hline 42 & S1 & Syringol & 32.74 & 0.057 & 0.059 \\
\hline 49 & $\mathrm{~S} 2$ & 4-Methylsyringol & 35.41 & 0.060 & 0.056 \\
\hline 53 & S3 & 4-Ethylsyringol & 37.49 & 0.010 & 0.010 \\
\hline 55 & S4 & 4-Vinylsyringol & 38.79 & 0.071 & 0.008 \\
\hline 62 & S5 & 4-Ethenylsyringol & 39.64 & 0.016 & 0.017 \\
\hline 71 & S6 & 4-(1-Propenyl)-2,6-dimethoxyphenol ( $Z$ & 40.99 & 0.013 & 0.011 \\
\hline 80 & S7 & $\begin{array}{l}\text { 4-(1-Propenyl)-2,6-dimethoxyphenol } \\
(E)\end{array}$ & 42.57 & 0.059 & 0.046 \\
\hline 95 & S8 & Syringaldehyde & 47.28 & 0.036 & 0.036 \\
\hline 110 & S9 & Sinapaldehyde & 55.95 & 0.011 & 0.010 \\
\hline & & & TOTAL & $\mathbf{0 . 3 3 3}$ & 0.253 \\
\hline
\end{tabular}


Table SI-4J. Concentrations of sugars and anhydrosugars in Oil B before and after $40 \mathrm{~h}$ of aging at $80^{\circ} \mathrm{C}$.

\begin{tabular}{|c|c|c|c|c|c|}
\hline 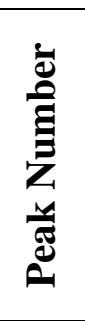 & 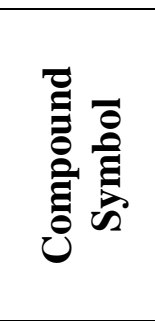 & Compound Name & 氖 & 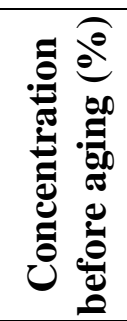 & 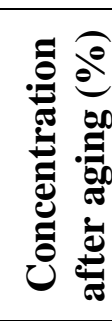 \\
\hline 70 & H1 & Galactosan & 40.78 & 0.254 & 0.203 \\
\hline 73 & $\mathrm{H} 2$ & Mannosan & 41.35 & 1.226 & 1.011 \\
\hline 75 & H3 & Levoglucosan & 41.99 & 3.852 & 3.847 \\
\hline 59 & $\mathrm{H} 4(\mathrm{a}, \mathrm{f})$ & $\mathrm{H} 4(\mathrm{a}, \mathrm{f})$ alpha arabinofuranose & 39.53 & 0.011 & 0.124 \\
\hline 61 & $\mathrm{H} 4(\mathrm{a}, \mathrm{p})$ & H4(a,p) alpha arabinopyranose & 39.60 & 0.005 & 0.042 \\
\hline 72 & $\mathrm{H} 4(\mathrm{~b}, \mathrm{f})$ & $\mathrm{H} 4(\mathrm{~b}, \mathrm{f})$ beta arabinofuranose & 41.24 & 0.016 & 0.091 \\
\hline 67 & $\mathrm{H} 4(\mathrm{~b}, \mathrm{p})$ & H4(b,p) beta arabinopyranose & 40.43 & 0.004 & 0.063 \\
\hline 64 & $\mathrm{H} 5(\mathrm{a}, \mathrm{f})$ & H5(a,f) alpha xylofuranose & 39.90 & 0.043 & 0.085 \\
\hline 77 & H5(a,p) & H5(a,p) alpha xylopyranose & 42.22 & 0.029 & 0.142 \\
\hline 66 & $\mathrm{H} 5(\mathrm{~b}, \mathrm{f})$ & H5(b,f) beta xylofuranose & 40.27 & 0.020 & 0.087 \\
\hline 85 & $\mathrm{H} 5(\mathrm{~b}, \mathrm{p})$ & H5(b,p) beta xylopyranose & 43.62 & 0.026 & 0.152 \\
\hline 94 & H6(a,p) & H6(a,p) alpha-glucopyranose & 46.89 & 0.033 & 0.117 \\
\hline 100 & H6(b,p) & H6(b,p) beta-glucopyranose & 49.19 & 0.019 & 0.110 \\
\hline 107 & $\mathrm{H} 7$ & H7, Cellobiosan & 53.92 & 0.226 & 0.225 \\
\hline 116 & $\mathrm{H} 8$ & H8, Cellobiose & 63.71 & 0.884 & 1.209 \\
\hline 44 & h1 & Unknown monosaccharide, furanose, h1 & 32.92 & 0.141 & 0.006 \\
\hline 74 & h2 & Unknown monosaccharide, furanose, h2 & 41.68 & 0.070 & 0.065 \\
\hline 79 & h3 & Unknown monosaccharide, furanose, h3 & 42.44 & 0.268 & 0.037 \\
\hline 81 & h4 & Unknown monosaccharide, furanose, h4 & 42.92 & 0.101 & 0.101 \\
\hline 82 & h5 & Unknown monosaccharide, furanose, h5 & 43.22 & 0.074 & 0.000 \\
\hline 83 & h6 & Unknown monosaccharide, furanose, h6 & 43.33 & 0.162 & 0.003 \\
\hline 86 & h7 & Unknown monosaccharide, furanose, h7 & 43.93 & 0.205 & 0.011 \\
\hline 92 & $\mathrm{~h} 8$ & Unknown monosaccharide, furanose, h8 & 45.83 & 0.168 & 0.167 \\
\hline 98 & h9 & Unknown disaccharide, $\mathrm{h} 9$ & 48.62 & 0.080 & 0.065 \\
\hline 104 & h10 & Unknown disaccharide, $\mathrm{h} 10$ & 51.21 & 0.143 & 0.132 \\
\hline 106 & h11 & Unknown disaccharide, $\mathrm{h} 11$ & 53.58 & 0.096 & 0.069 \\
\hline 115 & h12 & Unknown disaccharide, h12 & 63.26 & 0.338 & 0.153 \\
\hline 117 & h13 & Unknown disaccharide, $\mathrm{h} 13$ & 63.97 & 0.395 & 0.459 \\
\hline 118 & h14 & Unknown disaccharide, h14 & 64.56 & 0.289 & 0.279 \\
\hline \multicolumn{4}{|c|}{ TOTAL } & 9.179 & 9.054 \\
\hline
\end{tabular}


Table SI-4K. Concentrations of resin acids in Oil B before and after $40 \mathrm{~h}$ of aging at $80{ }^{\circ} \mathrm{C}$.

\begin{tabular}{|c|c|c|c|c|c|}
\hline 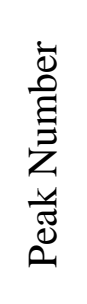 & 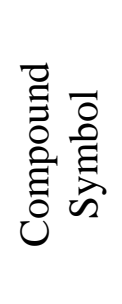 & Compound Name & 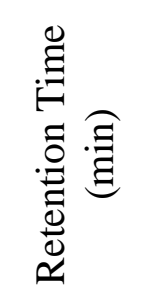 & 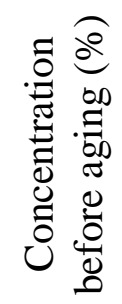 & 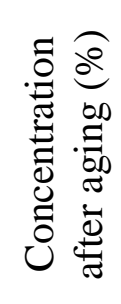 \\
\hline 111 & $\mathrm{~J} 1$ & Isopimaric acid & 56.69 & 0.352 & 0.361 \\
\hline 112 & $\mathrm{~J} 2$ & Dehydroabeitic acid & 57.71 & 0.818 & 0.796 \\
\hline 113 & $\mathrm{~J} 3$ & Abeitic acid & 58.37 & 0.193 & 0.153 \\
\hline & & & TOTAL & 0.454 & 0.437 \\
\hline
\end{tabular}

Table SI-4L. Concentrations of stilbenes in Oil B before and after $40 \mathrm{~h}$ of aging at $80{ }^{\circ} \mathrm{C}$.

\begin{tabular}{|c|c|c|c|c|c|}
\hline 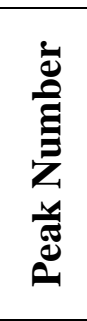 & 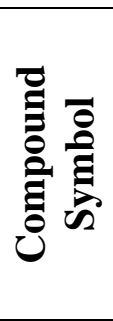 & Compound Name & 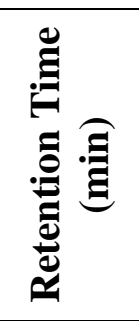 & 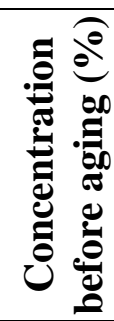 & 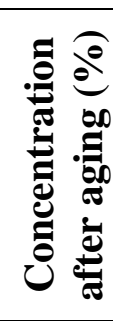 \\
\hline 101 & K1a & Methoxy/hydroxy stilbene (Z) & 50.45 & 0.010 & 0.002 \\
\hline 109 & K1b & Methoxy/hydroxy stilbene (E) & 55.94 & 0.046 & 0.006 \\
\hline 114 & $\mathrm{~K} 2 \mathrm{a}$ & Dimethoxy-dihydroxy stilbene $(Z)$ & 59.08 & 0.055 & 0.050 \\
\hline 119 & $\mathrm{~K} 2 \mathrm{~b}$ & Dimethoxy-dihydroxy stilbene (E) & 65.33 & 0.278 & 0.135 \\
\hline & & & TOTAI & 0.097 & 0.048 \\
\hline
\end{tabular}

Table SI-4M. Concentrations of others in Oil B before and after $40 \mathrm{~h}$ of aging at $80{ }^{\circ} \mathrm{C}$.

\begin{tabular}{|c|c|c|c|c|c|}
\hline 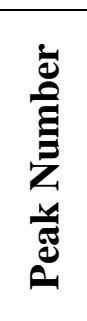 & 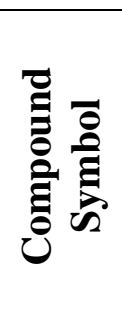 & Compound Name & 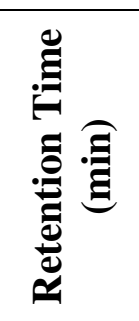 & 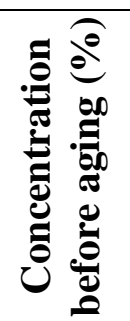 & 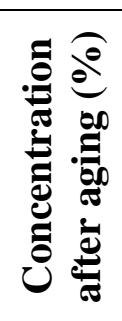 \\
\hline 122 & L1 & Shonanin & 66.84 & 0.196 & 0.177 \\
\hline 120 & 11 & Unknown MW $=576$ & 65.82 & 0.036 & 0.031 \\
\hline 121 & 12 & Unknown MW = 576 & 65.94 & 0.022 & 0.009 \\
\hline 123 & 13 & Unknown MW = 518 & 68.73 & 0.055 & 0.066 \\
\hline & & & TOTAI & 0.077 & 0.071 \\
\hline
\end{tabular}


Table SI-5. Summary of exact mass measurements for the putative trihydroxybenzene dimer, detected as the peracetylated form at $30.9 \mathrm{~min}$ in the chip-nanoESI-Q-TOF MS/MS analysis of Oil B.

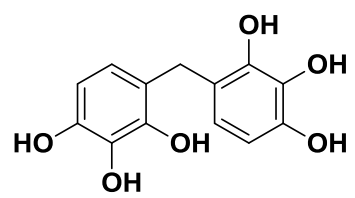

\begin{tabular}{|c|c|c|c|c|c|}
\hline $\begin{array}{c}\text { Measured } \\
{[\mathbf{M}+\mathbf{H}]^{\mathbf{+ a}}}\end{array}$ & $\begin{array}{c}\text { Predicted } \\
{[\mathbf{M + H}]^{+}}\end{array}$ & $\begin{array}{c}\text { Error } \\
(\mathbf{p p m})\end{array}$ & $\begin{array}{c}\text { Molecular } \\
\text { Formula }^{\mathbf{a}}\end{array}$ & $\begin{array}{c}\text { \# of Hydroxyl } \\
\text { Groups }^{\mathbf{b}}\end{array}$ & $\begin{array}{c}\text { Molecular } \\
\text { Formula }^{\mathbf{c}}\end{array}$ \\
\hline 517.1347 & 517.1341 & 1.2 & $\mathrm{C}_{25} \mathrm{H}_{24} \mathrm{O}_{12}$ & 6 & $\mathrm{C}_{13} \mathrm{H}_{12} \mathrm{O}_{6}$ \\
\hline
\end{tabular}

${ }^{\mathrm{a}}$ For the peracetylated product; ${ }^{\mathrm{b}}$ Established by the analysis of the trideuteroacetylated product; ${ }^{\mathrm{c}}$ Formula of the underivatized product. 